\title{
Expression of an alternatively spliced variant of SORL 1 in neuronal dendrites is decreased in patients with Alzheimer's disease
}

\author{
Giulia Monti', Mads Kjolby' ${ }^{1}$ Anne Mette G. Jensen', Mariet Allen², Juliane Reiche, Peter L. Møller', \\ Raquel Comaposada-Baró', Bartlomiej E. Zolkowski' , Cármen Vieira', Margarita Melnikova Jørgensen ${ }^{4,5}$, \\ Ida E. Holm ${ }^{4,5}$, Paul N. Valdmanis ${ }^{6}$, Niels Wellner ${ }^{1}$, Christian B. Vægter ${ }^{1}$, Sarah J. Lincoln ${ }^{2}$, Anders Nykjær ${ }^{1,7}$, \\ Nilüfer Ertekin-Taner ${ }^{2,8}$, Jessica E. Young ${ }^{9}$, Mette Nyegaard ${ }^{1}$ and Olav M. Andersen ${ }^{1,7^{*}}$ (D)
}

\begin{abstract}
SORL1 is strongly associated with both sporadic and familial forms of Alzheimer's disease (AD), but a lack of information about alternatively spliced transcripts currently limits our understanding of the role of SORL1 in AD. Here, we describe a SORL1 transcript (SORL 1-38b) characterized by inclusion of a novel exon (E38b) that encodes a truncated protein. We identified E38b-containing transcripts in several brain regions, with the highest expression in the cerebellum and showed that SORL1-38b is largely located in neuronal dendrites, which is in contrast to the somatic distribution of transcripts encoding the full-length SORLA protein (SORL1-fl). SORL1-38b transcript levels were significantly reduced in $A D$ cerebellum in three independent cohorts of postmortem brains, whereas no changes were observed for SORL 1-fl. A trend of lower 38b transcript level in cerebellum was found for individuals carrying the risk variant at rs2282649 (known as SNP24), although not reaching statistical significance. These findings suggest synaptic functions for SORL1-38b in the brain, uncovering novel aspects of SORL1 that can be further explored in AD research.
\end{abstract}

Keywords: Alzheimer's disease, SORLA, SORL 1, Alternative splicing, Dendritic transcript

\section{Introduction}

The SORL1 gene encodes the protein SORLA and is associated with Alzheimer's disease (AD) [1]. Recent burden analyses of ultra-rare variants through exome sequencing have found an excess of loss-of-function variants in AD cases, suggesting haploinsufficiency of SORL1 as a pathogenic mechanism in some patients [17, 44]. More recently, SORL1 missense variants in familial AD have also been reported [11, 29, 32], but the functional consequences of these variants are unknown.

*Correspondence: o.andersen@biomed.au.dk

1 Department of Biomedicine, Aarhus University, Høegh-Guldbergs Gade 10, 8000 Aarhus C, Denmark

Full list of author information is available at the end of the article
Previous studies have established SORLA as a sorting receptor for the amyloid precursor protein (APP) [1, 2]. This function depends on the physical contact between the extracellular parts of SORLA and APP, and the ability of the cytoplasmic tail of SORLA to form complexes with intracellular trafficking molecules including the retromer complex [15, 39]. Apart from its role as an APP trafficking determinant, very limited information exists about SORLA neuronal functions. There is therefore an increasing need to better understand the function of SORLA in the brain.

Alternative splicing (AS) is an essential process significantly involved in the expansion of the transcriptome and protein diversity. As another result of AS, transcripts from the same gene can also have different $3^{\prime}$ untranslated regions ( $3^{\prime}$ UTRs) and/or contain target motifs for original author(s) and the source, provide a link to the Creative Commons licence, and indicate if changes were made. The images or other third party material in this article are included in the article's Creative Commons licence, unless indicated otherwise in a credit line to the material. If material is not included in the article's Creative Commons licence and your intended use is not permitted by statutory regulation or exceeds the permitted use, you will need to obtain permission directly from the copyright holder. To view a copy of this licence, visit http://creativecommons.org/licenses/by/4.0/. The Creative Commons Public Domain Dedication waiver (http://creativeco mmons.org/publicdomain/zero/1.0/) applies to the data made available in this article, unless otherwise stated in a credit line to the data. 
RNA binding proteins within exonic sequences, responsible for distinct neuronal trafficking of transcript isoforms to axons or dendrites where they may be locally translated [12, 24, 43]. Recently, emerging evidence links AS with the maintenance of neuronal homeostasis [34], and associations between AS and AD have been reported [35]. For this reason, increased attention is directed towards AS of genes involved in neurodegenerative and neuropsychiatric diseases.

Although transcripts from $~ 95 \%$ of all human multiexon genes undergo AS [31], surprisingly little is known about the biological relevance for AS of SORL1. Due to an increasing number of putative transcripts annotated in databases, it is key to conduct experimental investigations to discriminate between functional transcripts and products derived by transcriptional noise. Here, we provide a detailed characterization of an alternatively spliced SORL1 transcript. Inclusion of a hitherto undescribed exon leads to transcripts that can be translated into a truncated receptor lacking its transmembrane and cytoplasmic domains, pointing towards a function unrelated to sorting of cargoes including APP. Using brain samples from three independent cohorts, we found decreased transcript expression of this truncating SORL1 isoform in AD patients, and identified enriched expression in neuronal dendrites suggesting a role of this novel isoform in synaptic plasticity, known to be impaired in AD.

\section{Materials and methods}

\section{Human samples}

We used biological material from four different sources: total RNA acquired from ClonTech, and human postmortem brain tissues from three different brain banks; the Netherlands Brain Bank (NBB), Mayo Clinic (Mayo), and University of Washington (UW).
From ClonTech, we obtained total RNA from brain, spinal cord, bone marrow, liver, heart, lung, trachea, kidney, adrenal gland, salivary gland, thyroid gland, thymus, skeletal muscle, colon, prostate, testis, placenta, and uterus.

From $N B B$, postmortem cerebella $(\mathrm{n}=3 \mathrm{AD} ; \mathrm{n}=3$ non-AD), hippocampus ( $\mathrm{n}=3$ non-AD) and entorhinal cortex $(\mathrm{n}=3$ non-AD) samples were obtained for in situ hybridization (ISH) (Table 1). NBB procedures have been approved by the ethics committee at the Vrije University Medical Center, Amsterdam, NL. The materials were donated to the bank on the basis of signed informed consent with the restriction of research purposes only. Diagnosis of AD was made by neuropsychological testing, followed by autoptic histopathological confirmation with disease propagation described by Braak staging. Following autopsy, cerebellum was dissected at the level of dentate nucleus, fixed in $4 \%$ paraformaldehyde and the tissue was embedded in paraffin blocks.

From Mayo Clinic, postmortem brain samples $(\mathrm{n}=25$ $\mathrm{AD} ; \mathrm{n}=25$ non-AD) were obtained for qPCR as previously described [5] and eQTL analysis (Table 2). Briefly, non-AD control samples had a Braak score of 3.0 or less and lacked any other major pathologic diagnoses; $\mathrm{AD}$ patients had a Braak score of $\geq 4.0$ and a definite diagnosis according to the NINCDS-ADRDA criteria [27]. Individuals were age matched across diagnosis groups ( \pm 1 year), were older than 60 years at time of death and $48 \%$ of each diagnosis group were male. This study was approved by the appropriate institutional review board.

From $U W$, postmortem cerebella tissue $(\mathrm{n}=14 \mathrm{AD}$; $\mathrm{n}=6$ non-AD) were selected for qPCR based on SNP24 genotype (Table 3 ).

Table 1. Netherlands Brain Bank samples data

\begin{tabular}{lllllllll}
\hline Region & Diagnosis & Sex & Age & PMD & pH & Weight & ApoE & Braak stage \\
\hline Cerebellum & Non-AD & M & 72 & $07: 25$ & 6.61 & 1370 & $3 / 3$ & 0 \\
Cerebellum & Non-AD & M & 69 & $05: 55$ & 6.41 & 1450 & $3 / 3$ & 0 \\
Cerebellum & Non-AD & M & 62 & $09: 35$ & 6.58 & 1163 & $3 / 3$ & 0 \\
Cerebellum & AD & M & 75 & $04: 20$ & 6.91 & 1325 & $3 / 3$ & 6 \\
Cerebellum & AD & M & 58 & $05: 10$ & 6.99 & 1180 & $3 / 3$ & 6 \\
Cerebellum & AD & M & 69 & $05: 30$ & 6.18 & 1086 & $3 / 3$ & 6 \\
Hippocampus & Non-AD & M & 91 & $08: 00$ & 6.26 & 1188 & $3 / 3$ & 1 \\
Hippocampus & Non-AD & M & 79 & $05: 45$ & 6.38 & 1361 & $3 / 3$ & 2 \\
Hippocampus & Non-AD & M & 96 & $04: 10$ & 6.09 & 1250 & $3 / 3$ & 4 \\
Amygdala/EC & Non-AD & M & 81 & $07: 55$ & 6.23 & 1194 & $3 / 3$ & 2 \\
Amygdala/EC & Non-AD & M & 78 & $<17: 40$ & 6.52 & 1125 & $3 / 3$ & 1 \\
Amygdala/EC & Non-AD & M & 79 & $05: 45$ & 6.38 & 1361 & $3 / 3$ & 2 \\
\hline
\end{tabular}

PMD post-mortem delay 
Table 2 Mayo Clinic cohort data

\begin{tabular}{|c|c|c|c|c|c|}
\hline Sample id & Diagnosis & Age at death & Gender & RIN & $\begin{array}{l}\text { GENOTYPE } \\
\text { rs2282649 }\end{array}$ \\
\hline 14546_CER & Non-AD & 83 & M & 8.3 & $\mathrm{C} / \mathrm{C}$ \\
\hline 14548_CER & Non-AD & 83 & M & 8.5 & $\mathrm{C} / \mathrm{C}$ \\
\hline 14549_CER & Non-AD & 63 & M & 8.8 & $\mathrm{C} / \mathrm{C}$ \\
\hline 14551_CER & Non-AD & 80 & $\mathrm{~F}$ & 9.3 & $\mathrm{C} / \mathrm{C}$ \\
\hline 14556_CER & Non-AD & 72 & $\mathrm{~F}$ & 8.9 & $\mathrm{C} / \mathrm{T}$ \\
\hline 7101_CER & Non-AD & 79 & M & 7.3 & na \\
\hline 7104_CER & Non-AD & 78 & F & 8.6 & na \\
\hline 14543_CER & Non-AD & 80 & M & 6.6 & $\mathrm{C} / \mathrm{C}$ \\
\hline 14562_CER & Non-AD & 76 & M & 8.1 & $C / T$ \\
\hline 1926_CER & Non-AD & 88 & $\mathrm{~F}$ & 7.7 & $\mathrm{C} / \mathrm{C}$ \\
\hline 1936_CER & Non-AD & 89 & $\mathrm{~F}$ & 6.6 & $C / T$ \\
\hline 1955_CER & Non-AD & 87 & F & 8.3 & $\mathrm{C} / \mathrm{C}$ \\
\hline 1960_CER & Non-AD & 97 & $\mathrm{~F}$ & 7.6 & $\mathrm{C} / \mathrm{C}$ \\
\hline 1963_CER & Non-AD & 94 & M & 9.1 & $C / T$ \\
\hline 1964_CER & Non-AD & 92 & M & 9.2 & $\mathrm{C} / \mathrm{C}$ \\
\hline 14557_CER & Non-AD & 81 & M & 8.1 & $\mathrm{C} / \mathrm{T}$ \\
\hline 1919_CER & Non-AD & 87 & $\mathrm{~F}$ & 9.2 & $C / T$ \\
\hline 1933_CER & Non-AD & 98 & F & 7.8 & $\mathrm{C} / \mathrm{C}$ \\
\hline 1934_CER & Non-AD & 89 & M & 9.7 & $\mathrm{C} / \mathrm{T}$ \\
\hline 1937_CER & Non-AD & 86 & $\mathrm{~F}$ & 8.5 & $\mathrm{C} / \mathrm{C}$ \\
\hline 1940_CER & Non-AD & 96 & M & 7.5 & $\mathrm{C} / \mathrm{C}$ \\
\hline 1944_CER & Non-AD & 98 & $\mathrm{~F}$ & 7.1 & $\mathrm{C} / \mathrm{C}$ \\
\hline 1945_CER & Non-AD & 90 & M & 8.9 & $\mathrm{C} / \mathrm{C}$ \\
\hline 1950_CER & Non-AD & 86 & F & 7.6 & $C / T$ \\
\hline 1952_CER & Non-AD & 92 & $\mathrm{~F}$ & 8.8 & $C / T$ \\
\hline 1058_CER & $A D$ & 95 & M & 7.5 & $\mathrm{C} / \mathrm{C}$ \\
\hline 1166_CER & $A D$ & 84 & M & 8.5 & $C / T$ \\
\hline 142_CER & $A D$ & 89 & $\mathrm{~F}$ & 9.2 & $\mathrm{C} / \mathrm{C}$ \\
\hline 747_CER & $A D$ & 62 & M & 8.9 & na \\
\hline 807_CER & $A D$ & 81 & M & 7.9 & na \\
\hline 851_CER & $A D$ & 87 & $\mathrm{~F}$ & 9.1 & $C / T$ \\
\hline 896_CER & $A D$ & 80 & $F$ & 9.1 & $\mathrm{C} / \mathrm{C}$ \\
\hline 1034_CER & $A D$ & 88 & F & 7.8 & $T / T$ \\
\hline 1046_CER & $A D$ & 72 & $\mathrm{~F}$ & 9.2 & $\mathrm{C} / \mathrm{C}$ \\
\hline 1052_CER & $A D$ & 91 & M & 8.4 & $C / T$ \\
\hline 1114_CER & $A D$ & 82 & M & 8.4 & $T / T$ \\
\hline 1169_CER & $A D$ & 92 & M & 8.5 & na \\
\hline 907_CER & $A D$ & 95 & $\mathrm{~F}$ & 7.6 & na \\
\hline 953_CER & $A D$ & 86 & F & 8.3 & na \\
\hline 720_CER & $A D$ & 75 & M & 8.1 & na \\
\hline 741_CER & $A D$ & 89 & M & 8.4 & na \\
\hline 790_CER & $A D$ & 86 & F & 7.5 & na \\
\hline 809_CER & $A D$ & 87 & $F$ & 8.4 & na \\
\hline 911_CER & $A D$ & 89 & $\mathrm{~F}$ & 7.3 & na \\
\hline 963_CER & $A D$ & 99 & F & 7.7 & na \\
\hline 990_CER & $A D$ & 79 & M & 6.7 & na \\
\hline 1101_CER & $A D$ & 78 & M & 7.6 & na \\
\hline 1146_CER & $A D$ & 100 & $\mathrm{~F}$ & 8.5 & $\mathrm{C} / \mathrm{C}$ \\
\hline 731_CER & $A D$ & 93 & M & 9.0 & $\mathrm{~T} / \mathrm{T}$ \\
\hline 966_CER & $A D$ & 79 & 2 & 8.8 & $C / T$ \\
\hline
\end{tabular}

Table 2 (continued)

RIN RNA integrity number

SORL1-38b transcript levels across different human tissues Total RNA from ClonTech was converted to cDNA using $1 \mu \mathrm{g}$ of RNA and High Capacity RNA-to-cDNA kit (Cat N. \#4387406, Applied Biosystems, Europe). PCR was per-

Table 3 University of Washington cohort data

\begin{tabular}{|c|c|c|c|c|}
\hline Sample id & Diagnosis & Age at death & Gender & $\begin{array}{l}\text { GENOTYPE } \\
\text { rs2282649 }\end{array}$ \\
\hline 1706_CER & Non-AD & 76 & $\mathrm{~F}$ & $\mathrm{~T} / \mathrm{T}$ \\
\hline 1661_CER & Non-AD & 92 & $\mathrm{~F}$ & $\mathrm{C} / \mathrm{T}$ \\
\hline 1776_CER & Non-AD & 98 & $\mathrm{~F}$ & $\mathrm{C} / \mathrm{T}$ \\
\hline 1750_CER & Non-AD & 78 & M & $\mathrm{C} / \mathrm{C}$ \\
\hline 1792_CER & Non-AD & 93 & $\mathrm{~F}$ & $\mathrm{C} / \mathrm{C}$ \\
\hline 1814_CER & Non-AD & 86 & $\mathrm{~F}$ & $\mathrm{C} / \mathrm{C}$ \\
\hline 1798_CER & $A D$ & 93 & $\mathrm{~F}$ & $\mathrm{~T} / \mathrm{T}$ \\
\hline 1744_CER & $A D$ & 93 & $\mathrm{~F}$ & $\mathrm{~T} / \mathrm{T}$ \\
\hline 1745_CER & $A D$ & 82 & $\mathrm{~F}$ & $\mathrm{~T} / \mathrm{T}$ \\
\hline 1784_CER & $A D$ & $90+$ & $\mathrm{F}$ & $\mathrm{T} / \mathrm{T}$ \\
\hline 1785_CER & $A D$ & 93 & M & $\mathrm{T} / \mathrm{T}$ \\
\hline 1815_CER & $A D$ & 67 & $\mathrm{~F}$ & $\mathrm{~T} / \mathrm{T}$ \\
\hline 1682_CER & $A D$ & 100 & $\mathrm{~F}$ & $\mathrm{C} / \mathrm{T}$ \\
\hline 1733_CER & $A D$ & 92 & $\mathrm{~F}$ & $\mathrm{C} / \mathrm{T}$ \\
\hline 1746_CER & $A D$ & 69 & M & $\mathrm{C} / \mathrm{T}$ \\
\hline 1771_CER & $A D$ & 98 & $\mathrm{~F}$ & $\mathrm{C} / \mathrm{T}$ \\
\hline 1806_CER & $A D$ & 65 & M & $\mathrm{C} / \mathrm{T}$ \\
\hline 1662_CER & $A D$ & 82 & $\mathrm{~F}$ & $\mathrm{C} / \mathrm{C}$ \\
\hline 1681_CER & $A D$ & 74 & $\mathrm{~F}$ & $\mathrm{C} / \mathrm{C}$ \\
\hline 1707_CER & $A D$ & 90 & M & $\mathrm{C} / \mathrm{C}$ \\
\hline 1804_CER & $A D$ & 101 & $\mathrm{~F}$ & $\mathrm{C} / \mathrm{C}$ \\
\hline
\end{tabular}

formed using Herculase II Fusion DNA Polymerase (Cat N. \#600675, Agilent, Europe) on a Veriti Thermal Cycler (Applied Biosystems) in the presence of 3\% DMSO with the following optimized protocol: $95^{\circ} \mathrm{C}$ for $2 \mathrm{~min}$, followed by 35 cycles of amplification $\left(95{ }^{\circ} \mathrm{C}\right.$ for $20 \mathrm{~s}$, $52{ }^{\circ} \mathrm{C}$ for $20 \mathrm{~s}$ and $72{ }^{\circ} \mathrm{C}$ for $30 \mathrm{~s}$ ) and extension at $72{ }^{\circ} \mathrm{C}$ for $3 \mathrm{~min}$. SORL1-38B was amplified using primers in exon 35 and 38b. Primer sequences were SORL1-Ex35fw: 5'-GGCACACAACACCAATGACT, and SORL1Ex38B-rev; 5'-TGCTCTTCCAACATCCCTTCT. Water was used as negative control. RT-PCR for the analysis of splicing events downstream of E38b was performed using Titanium One-Step RT-PCR Kit (Cat N. 639504, ClonTech) on total RNA from human cerebellum (Cat N. 636535, ClonTech) with primers located in exons 38b and 40. Primer were SORL1-Ex38b-fw: $5^{\prime}$-TGACCACAC ATACCAAGAAGGG and SORL1-Ex40-rev: 5'-TGC 
TTCCTCGGAAGTTCAAAGT. The refined analysis of transcript levels across tissue was performed using qPCR on the same samples and protocol for cDNA synthesis. Each cDNA was tested with a custom Taqman probe for detection of SORL1-38b (SORLA_EX_37-38B, AssayID AJBJXYH, Cat N. 4441114) (Applied Biosystems), and pre-designed probes for SORL1 exon 3-4 (Hs00268342 $\mathrm{m} 1$ ). Three household genes were analysed: HPRT1 (Hs02800695_m1), TFRC (Hs00951083_m1), and TXNL1 (Hs00355488_m1). Singleplex PCR was performed in 96-well plates LightCycler 480 System (Roche) with the following conditions: $95{ }^{\circ} \mathrm{C}$ for $10 \mathrm{~s}, 45$ cycles of amplification $\left(95^{\circ} \mathrm{C}\right.$ for $10 \mathrm{~s}, 60^{\circ} \mathrm{C}$ for $30 \mathrm{~s}, 72{ }^{\circ} \mathrm{C}$ for $\left.1 \mathrm{~s}\right)$, and $72{ }^{\circ} \mathrm{C}$ for $1 \mathrm{~s}$. The efficiency and dynamic range for each qPCR assay was established using a serial dilution, and confirmed to be above $92 \%$ for all assays.

\section{Generation of SORL1-38b expression construct}

The small fragment in human SORL1 from exon 37 to exon 38B was synthesized in pEX-A2 vector (MWG, Eurofins, Germany) including the native NdeI site in exon 37 and a Nsil site at the $3^{\prime}$ end. The fragment was cloned using NdeI and NsiI into the human SORL1 in pGEM EASY vector [19], and was subsequently subcloned to a pcDNA3.1 (Zeo) vector for cell studies. Site directed mutagenesis was used for introducing mutations leading to Cys to Ala substitutions at position 1 and 13 of the tail encoded by E38b using Quickchange XL SiteDirected Mutagenesis (Cat N. 200516) (Agilent). The same approach was applied for introducing stop codons after each 3Fn domain in a pcDNA3.1 plasmid containing the cDNA for human SORL1.

\section{Antibodies}

A polyclonal antibody against SORLA-38b was generated in rabbit by peptide immunization against the unique c-terminal VCFKTLTTHTKKGCWKSNQIHVP encoded by E38b (Aldevron, Germany). The immunogen CFKTLTTHTKKG-Abu-WKSNQIHVP was synthesized, where the C-terminal cysteine was substituted with aminobutyric acid (Abu). Keyhole limpet hemocyanin was conjugated to the immunogen for immunization. The antibody was subsequently purified from serum, and its specificity was assessed by Western blot (WB) analysis.

Mouse monoclonal LR11 antibody was purchased from R\&D systems (Cat N. 611860), and rabbit polyclonal antisol SORLA (IgG 5387) and anti-tail SORLA were from colleagues at Aarhus University.

\section{Cell culture}

HEK-293 cells were cultured in Dulbecco's Modified Eagle's Medium (DMEM, Sigma) supplemented with 10\% fetal bovine serum and penicillin/streptomycin $(\mathrm{P} / \mathrm{S})$ in a humidified $5 \% \mathrm{CO}_{2}$ incubator at $37{ }^{\circ} \mathrm{C}$. Fugene 6 Transfection Reagent kit (Cat N. E2691, Promega) was used to transfect cells with SORL1-38b or SORL1- $f$ constructs according to manufacturer's instructions. After $24 \mathrm{~h}$, medium with zeocin was added to the transfected cells for selection and generation of a stable cell line expressing SORL1-38b construct. CHO cells were cultured in HyClone medium (Sigma) supplemented with 5\% $\mathrm{P} / \mathrm{S}$, and were transfected with SORL1 constructs using Fugene kit.

\section{Pulse-chase analysis}

Cells were incubated in methionine- and cysteine-free medium (Cat N. D0422, Sigma) supplemented with glutamax and $2 \%$ dialyzed FBS (Gibco) for $15 \mathrm{~min}$ at $37^{\circ} \mathrm{C}$, prior to biolabeling with $\left[{ }^{35} \mathrm{~S}\right]$ Promix (Amersham Biosciences) for $40 \mathrm{~min}$ at $37{ }^{\circ} \mathrm{C}$. Subsequently, cells were washed with phosphate-buffered saline (PBS), and both media and lysates were collected at different chase points. Cell lysates were immunoprecipitated at $4{ }^{\circ} \mathrm{C}$ overnight with GammaBind G-Sepharose beads (Amersham Biosciences) previously labeled with anti-sol-SorLA diluted in HyClone medium. Beads were then washed with PBS and proteins were resolved by standard SDS-PAGE and fluorography.

\section{Immunoblotting}

Transfected cells were lysed in lysis buffer containing protease inhibitor (Complete, Roche), and media were collected. Proteins were fractioned by $4-12 \%$ SDS-gel electrophoresis, and transferred to nitrocellulose membranes (Amersham). Membranes were blocked in blocking buffer (20\% TST buffer $(0.25 \mathrm{M}$ Tris-Base, $2.5 \mathrm{M}$ $\mathrm{NaCl}, 0.5 \%$ Tween $20 \mathrm{pH}$ 9), 2\% skimmed milk powder, $2 \%$ Tween 20) for $1 \mathrm{~h}$ at RT, and incubated at $4{ }^{\circ} \mathrm{C}$ overnight with mouse anti-LR11 (1:500), and rabbit pAb-38b (1:1000) primary antibodies. Incubation with HRP-conjugated anti-mouse $(1: 1500)$ or anti-rabbit $(1: 1500)$ secondary antibodies was performed at RT for $1 \mathrm{~h}$. Proteins were detected with SuperSignal West Femto Maximum Sensitivity Substrate (Thermo Fisher) and visualized with LAS-1000 (GE Healthcare).

\section{In situ hybridization}

From NBB, paraffin embedded human cerebellum, hippocampus and entorhinal cortex were cut to $5 \mu \mathrm{m}$ thick sections using a microtome. ISH was carried out by the BaseScope and BaseScope Duplex technologies (Advanced Cell Diagnostics) following manufacturer's instructions. Tissue hybridization was performed for $2 \mathrm{~h}$ at $40{ }^{\circ} \mathrm{C}$ (Hybez oven, ACDbio) with specific probes designed to span exon-exon junctions for recognition of 
SORL1-38b (E38E38b; E38bE39; E38bE40) and SORL1-fl transcripts (E38E39). In parallel, sections were hybridized with a PPIB probe (Cat N. 322975, ACDBio) as positive control. Chromogenic detection of specific hybridization was assessed with Fast Red dye (Cat N. 323600, ACDbio), and Fast Red-Green (Cat N. 323800, ACDBio). Nuclei counterstaining was made by incubation with Mayer's hematoxylin for $2 \mathrm{~min}$ at RT. Tissues were rinsed in deionized water and mounted with VectaMount permanent medium (Vector labs, Burlingame, CA). Slices were imaged with a Zeiss Apotome brightfield camera (Zeiss Apotome). Number of transcripts was manually quantified by counting punctate dots observed in PCs in the entire sections. Data were then plotted as mean number of transcripts per counting group (Group 0: 0 signal; Group I: 1 signal; Group II: 2-3 signals; Group III: $>4$ signals). For quantification of somatic and extra-somatic transcripts after BaseScope treatment, 25 random fields were chosen at 20X magnification, counting three PCs in each field in order to compare homogenous tissue areas.

\section{RNA-seq read alignment}

Two published datasets were queried for the presence of RNA-seq reads spanning the exon 38b splice junctions. We downloaded RNA sequencing alignment files generated from the cerebellum [33] along with frontal lobe, superior temporal gyrus and parahippocampal gyrus brain regions generated from the Mount Sinai Brain Bank [46]. We quantified the ratio of E38E38b versus E38E39 splice junctions reads. Reads spanning the E38E38b, E38bE39 and E38bE40 junctions collected from parahippocampal gyrus samples were overlaid onto the UCSC human genome browser.

\section{SORL1-38b differential expression in AD and non-AD samples}

The cDNA synthesis and qPCR analysis on the Mayo samples were conducted at Mayo Clinic. RNA was isolated from 50 cerebellum samples from the Mayo Clinic brain bank using TRIzol Reagent (Ambion Life Technology) followed by DNase RNA cleanup step using RNeasy (Qiagen). The quantity and quality of RNA samples were determined by the Agilent 2100 Bioanalyzer using Agilent RNA 6000 Nano Chip. All samples had an RNA integrity number (RIN) of $\geq 6.6$, with an equivalent mean RIN for both diagnosis groups (mean RIN AD $=8.26$, mean RIN controls $=8.24$ ). cDNA synthesis was performed with 1-2 $\mu \mathrm{g}$ of RNA using the High Capacity RNA-to-cDNA kit (Applied Biosystems cat. no. 4387406) according to the manufacturer's instructions. The samples were tested with a custom Taqman probe for detection of SORL1-38b, and three housekeeping genes: HPRT1, TFRC, and TXNL1. Four replicate measures were taken per sample for each of the four Taqman assays (16 measures per sample) on 384 well reaction plates using the QuantStudio ${ }^{\mathrm{TM}} 7$ Flex Real-Time PCR system and analysis software (Applied Biosystems, California, USA).

The cDNA synthesis and qPCR analysis on the UW samples were conducted at the University of Washington. From each sample, $1 \mu \mathrm{g}$ total cerebellum RNA was converted to cDNA (iScript, Biorad). SYBR Green qPCR was performed using Real-Time PCR Detection System (Bio$\mathrm{rad})$ with the following primers for SORL1-38b: E38bFw: 5'-GCGGTGACTAGTCGTGGAAT and E38b-Rev: 5'-TGCTCTTCCAACATCCCTTC. PCR products were amplified with the following conditions: $50^{\circ} \mathrm{C}$ for $2 \mathrm{~min}$, $95{ }^{\circ} \mathrm{C}$ for $20 \mathrm{~s}, 40$ cycles of amplification $\left(95^{\circ} \mathrm{C}\right.$ for $3 \mathrm{~s}$, $60{ }^{\circ} \mathrm{C}$ for $30 \mathrm{~s}$ ). Three housekeeping genes were used as controls: HPRT1, CYC1 and RPL13.

Each sample was run in triplicates, and water was included as negative control.

\section{Genotyping of SNP24}

Briefly, genomic DNA was prepared from cerebella samples from UW Neuropathology Core. DNA was genotyped for the presence of risk (T) or protective (C) allele at position rs2282649 by Sanger Sequencing (Genewiz). In total, 67 samples were genotyped and we observed $7 \mathrm{~T} / \mathrm{T}$ carriers (10.4\%), $29 \mathrm{C} / \mathrm{C}$ carriers (43.3\%), and 31 $\mathrm{C} / \mathrm{T}$ carriers $(46.3 \%)$. To obtain groups of equal sample size, we chose 7 samples of each genotype $(C / C, C / T$, and TT) for $38 \mathrm{~b}$ transcript analysis. One sample from the $\mathrm{T} / \mathrm{T}$ genotype group failed qPCR of SORL1-38b due to Ct values outside the dynamic range for the assay. Primers for PCR of amplicon containing SNP24 was FW: 5'-CTT TAGCTCATTCAGTATTCTTACTGTATG, and Rev: 5'-GAGCATTTCTTCTAATGCAGACATAC.

\section{Statistical analysis \\ In situ semi quantitative analysis}

An unpaired student's t test was performed in Graphpad Prism 5.0 to compare differences and determine statistical significance between $\mathrm{AD}$ and non-AD samples. A $p$ value below 0.05 was considered significant, indicated as $p<0.05\left(^{(*)}\right), p<0.01\left(^{(* *)}\right)$, and $p<0.001{ }^{(* * *)}$.

\section{Differential expression of $38 b$ transcript in $A D$ versus non- $A D$} Comparison of normalized SORL1-38b transcript levels between AD and non-AD samples in the Mayo and UW cohorts was performed in $\mathrm{R}$ version 3.6.1. First, a linear regression between deltaCT and diagnosis was performed, with RIN (only available for Mayo cohort), age at death, gender and plate ID as covariates. As no significant association was found for any of the covariates, 
a Wilcoxon rank sum test was subsequently used to compare $\mathrm{AD}$ versus non-AD samples. A meta-analysis of the two cohorts was performed using the metap $\mathrm{R}$ package (version 1.3). (Dewey M (2020). metap: meta-analysis of significance values).

\section{Association of SNP24 with AD}

Comparison of the risk allele frequency between $\mathrm{AD}$ and non-AD samples for SNP24 in the Mayo cohort was performed using Fisher's exact test. This test was not performed in the UW cohort, as samples were chosen based on genotype, so allele frequencies were not representative for $\mathrm{AD}$ cases and controls.

\section{Expression of $38 b$ transcript stratified on SNP genotype}

Whole genome-sequence data for 35 of the Mayo Clinic samples is available via the AD Knowledge Portal (https://adknowledgeportal.synapse.org, https://doi. org/10.7303/syn2580853). The AD Knowledge Portal is a platform for accessing data, analyses, and tools generated by the Accelerating Medicines Partnership (AMP-AD) Target Discovery Program and other National Institute on Aging (NIA)-supported programs to enable open-science practices and accelerate translational learning. The data, analyses and tools are shared early in the research cycle without a publication embargo on secondary use. Data is available for general research use according to the following requirements for data access and data attribution (https://adknowledgeportal.synapse.org/DataA ccess/Instructions). For access to content described in this manuscript see: https://www.synapse.org/\#!Synapse: syn11714444. Genotypes were extracted from the available VCF files using vcftools version 0.1.17.

To determine the effect of SNP24 on transcript levels, type I ANOVA was used to compare the three groups, $\mathrm{C} / \mathrm{C}, \mathrm{C} / \mathrm{T}$ and $\mathrm{T} / \mathrm{T}$, with diagnosis as covariate. Normal distribution was checked visually using qqplot and Shapiro-Wilk test. The eQTL analysis of 38b was performed on 35 samples from the Mayo cohort using the
MatrixEQTL package [41]. The cis-eQTL analysis was restricted to SNPs in the SORL1 gene $\pm 1 \mathrm{Mbp}$. Only SNPs with at least 5 and at most 65 alternate allele counts of the total 70 alleles were used. MatrixEQTL was run with modelLINEAR and disease status as covariate.

\section{Results}

\section{Identification of a novel primate-specific exon in SORL1}

The SORL1 gene on human chromosome 11 spans $>181 \mathrm{~kb}$ and contains at least 48 assigned exons [36]. The mRNA produced by assembling these known exons encodes the main form of SORLA referred to as the full-length receptor (SORLA-fl).

Here we validated the existence of a novel 118 bp exon located between exons 38 and 39, hereafter referred to as "exon 38b" (E38b) (Fig. 1a). A human tissue RNA library was screened by RT-PCR using an E38b specific primer pair (for exons $35 / 38 b$ ), revealing inclusion of this exon in transcripts from numerous tissues, including both fetal and adult brain. Several tissues did not express SORL1$38 b$ although they were positive for canonical SORL1 transcripts (identified by primer pair for exons $2 / 3$ ), indicating differential expression of the alternatively spliced transcript (Fig. 1b).

Sequencing of the RT-PCR product from brain identified a E38b-containing transcript encoding a protein with 22 new amino acids followed by a stop-codon (Fig. 1f). The resulting truncated receptor protein is hereafter termed SORLA-38b (Fig. 1c). Notably, the junction between exons 38 and 39 corresponds exactly to the boundary between the second and the third fibronectintype III (3Fn)-domains of SORLA (between residues 1747 and 1748). Alignment analysis of the genomic sequence across species confirmed E38b conservation from human to Pan Troglodytes, Macaca mulatta, and Gorilla gorilla genomes, whereas this exonic cassette is absent in nonprimate phylogeny.

The presence of the unique 22 residues allowed us to raise an antibody (pAb-38b, Fig. 1c, d) specifically

\footnotetext{
(See figure on next page.)

Fig. 1 A novel alternatively spliced SORL 1 transcript encodes a truncated receptor. a Schematic representation of the genomic region of SORL 1 including the novel exon 38b (red) located in close proximity to SNP24 within the $3^{\prime}$ risk haplotype ( $\left.1200 \mathrm{bp}\right)$. Primers for amplification of the 388 bp fragment between exon 35 and 38b are indicated with arrows. b RT-PCR showing specific expression of SORL 1-38b transcripts (E35-E38b) in only some human tissues, compared to ubiquitous expression of SORL1 transcripts containing exons 2 and 3 (E2-E3), and 18S ribosomal RNA (RN18S) as control. c WB analysis of lysates and media of cells transfected with cDNA encoding either SORLA-fl or SORLA-38b. The antibody pAb-38b specifically recognizes the truncated $188 \mathrm{kDa}$ SORLA-38b receptor, but not the SORLA-fl protein ( $250 \mathrm{kDa})$. $\mathbf{d}\left[{ }^{35} \mathrm{~S}\right]$-pulse-chase analysis on cells transfected with constructs encoding SORLA-fl (left) or SORLA-38b (right), with both isoforms mainly located in cell lysates. SORLA-fl goes from an immature (black arrowhead) to a mature (white arrowhead) form during the $8 \mathrm{~h}$ chase period, and the mature protein can be shed into the medium after $4 \mathrm{~h}$. Trace amounts of SORLA-38b is secreted as early as $30 \mathrm{~min}$ after radiolabeling, but can undergo endocytosis and be re-secreted in a mature form after $4 \mathrm{~h}$. e WB analysis for a panel of SORLA deletion constructs terminating after different 3Fn-domains in cell lysates (L) or medium $(\mathrm{M})$, showing how the presence of the fifth $3 \mathrm{Fn}$-domain (3Fn5) is responsible for cellular retention of soluble SORLA constructs. f Genomic sequence of E38b (in red) with flanking splice regions (in black), including translated amino acid sequence shown on top
} 
a

$$
\begin{gathered}
\text { genomic DNA SORL1 } \\
\text { SORL1-fl mRNA } \\
\text { SORL1-38b mRNA }
\end{gathered}
$$

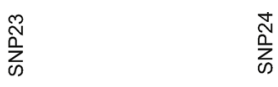

$\frac{1}{4}$

$2 \mathrm{~kb}$

$40 \quad 41$

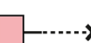

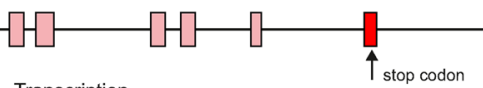

Transcription

Alternative splicing

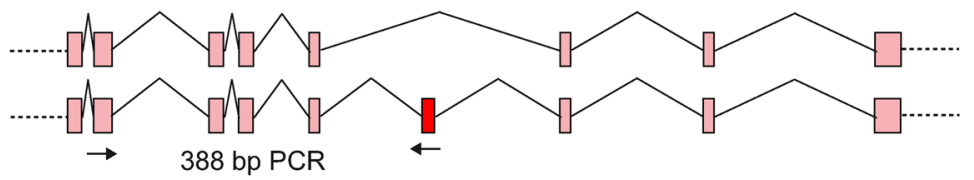

b

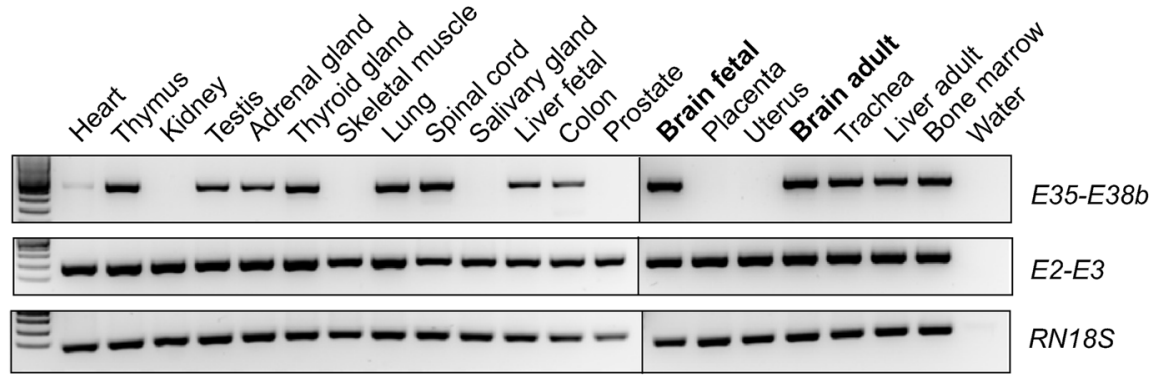

C

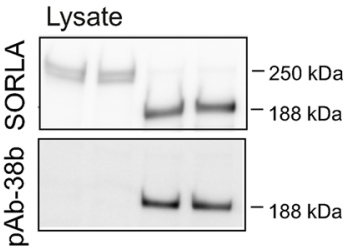

d

SORLA-fl

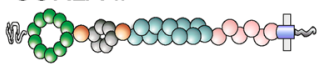

SORLA-38b

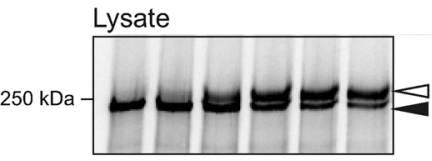

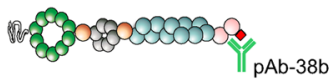

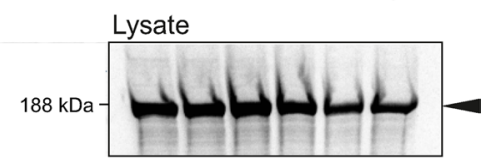

Medium

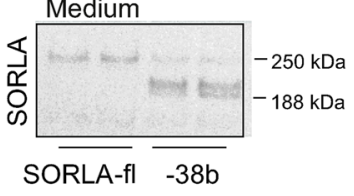

Medium

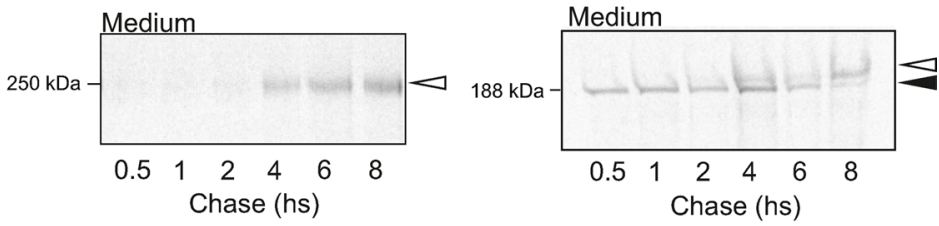

e

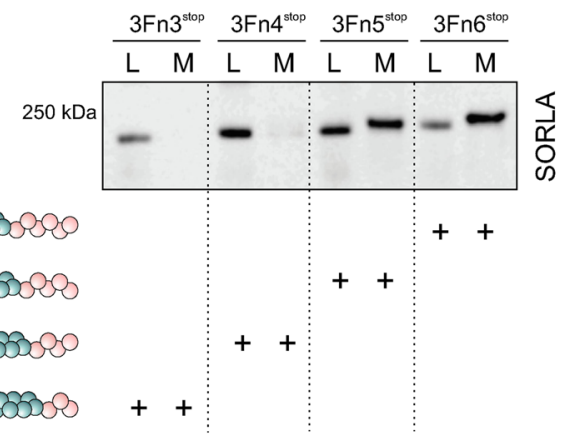

f $\begin{array}{rllll}{ }^{1747} \mathrm{Val} & \text { CYs } & \text { Phe Lys } & \text { Thr } & \text { Leu } \\ \text { TCTACAAAGTC TGT TTC AAA ACA } & \text { CTTG }\end{array}$ Thr Thr His Thr Lys Lys Gly Cys ACC ACA CAT ACC AAG AAG GGA TGI Trp Lys Ser Asn Gin Ile His Val Pro $* * *$

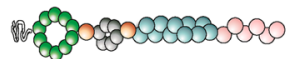

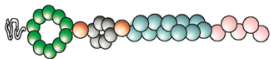

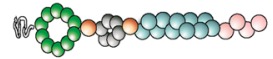

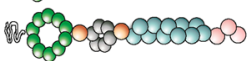


recognizing the unique C-terminal end of SORLA-38b, thereby enabling us to distinguish the truncated isoform from SORLA-fl, as demonstrated by WB analysis. As expected, only cells transfected with a cDNA for SORL1$38 b$, but not SORL1- $f l$, produced a product recognized by pAb-38b. Surprisingly, in this experiment only a limited fraction of SORLA-38b was secreted into the medium despite the lack of any transmembrane region, suggesting another mechanism of cell retention (Fig. 1c). A $\left[{ }^{35} \mathrm{~S}\right]$-pulse-chase experiment confirmed that SORLA38b stays mainly cell-associated (Fig. 1d).

To investigate why SORLA-38b is not efficiently secreted, we experimentally substituted each of two cysteine residues that are present in the unique SORLA$38 \mathrm{~b}$ sequence. We found that neither of the two residues-when mutated separately or simultaneously-had any effect on secretion (Additional file 1: Fig. S1a). As deletion of all 22 amino acids from the SORLA-38b isoform also did not affect secretion, we finally measured secretion of a panel of SORLA deletion constructs, demonstrating that the presence of the fifth 3Fn-domain is essential for secretion of the SORLA extracellular domain (Fig. 1e, Additional file 1: Fig. S1b). Although the exact molecular mechanism remains to be determined in detail, these data strongly suggest that the truncated SORLA-38b is structurally different compared to the fulllength SORLA receptor, in line with distinct unrelated functions.

Combined, these findings indicate tissue-specific expression of a novel SORL1 transcript, that can be translated into a truncated and stable SORLA-38b protein isoform with a function unrelated to the full-length receptor.

\section{High SORL1-38b expression in cerebellum}

We next measured the expression levels of SORL1-38b across multiple brain regions by RT-PCR. While expression was evident for all tested regions including the temporal lobe, hippocampus, frontal cortex and entorhinal cortex, a consistently higher expression was observed in the cerebellum, a region previously reported to express high levels of SORLA [5, 28, 30]. A follow-up qPCR analysis demonstrated 2.1-fold enrichment of SORL1-38b transcript in cerebellum compared to whole brain (i.e. $\Delta \Delta \mathrm{Ct}=-1.1$ ) (Fig. 2a, b). Accordingly, we focused our first analysis on cerebellum.

To determine in detail the histological distribution of SORL1-38b transcript, we employed BaseScope technology for the in situ detection of the splice variant and SORL1- $f$ in brain specimens (Fig. 2c). Whereas the probe for SORL1- $f$ evenly labelled all Purkinje cells (PCs) as well as many cells in the granule cell layer (GCL), the probe for SORL1-38b showed a restricted signal mainly within the Purkinje cell layer and clearly absent from the GCL (Fig. 2d). Detailed inspection of the cerebellar sections tested for SORL1 expression revealed a difference in the cellular distribution of the two probes $(p<0.0001)$. While the signal for SORL1-fl was predominantly (89\%) located in the soma of PCs, SORL1-38b was only partially present in the PC soma (46\%) with the majority of labelled transcripts located in the molecular layer where the dendritic tree of PCs extends (Fig. 2e, f).

These results pointed out PCs as the major source of production of E38b-containing SORL1 transcripts in the cerebellum, and showed a regional difference between transcripts encoding SORLA-fl and SORLA-38b.

\section{Exon $38 \mathrm{~b}$ determines neuronal dendritic targeting}

As the $3^{\prime}$ UTR of transcripts is often involved in mRNA transportation in neurons [12, 43], we next applied a protocol for analyzing the $3^{\prime}$ end of the transcript by $3^{\prime}$ RACE PCR using RNA from human cerebellum. We found that both SORL1-38b and SORL1-fl end with exon 48 suggesting identical poly-adenylation for the two isoforms (Additional file 2: Fig. S2). We further examined the immediate $3^{\prime}$ region downstream of the stop-codon (introduced by inclusion of E38b) by RT-PCR using RNA from four different brain regions as well as whole brain. This analysis demonstrated the presence of two distinct E38b-containing transcripts, most notably in the cerebellum (Fig. 3a). Sequencing of these two PCR products identified a second alternative splice event where E38b can join directly to either the downstream exon 39 or to exon 40 by exclusion of exon 39 .

Because transcripts for SORL1- $f$ contain exon 39 and mainly locate to the neuronal soma, we hypothesized a model where exon 39 would dictate somatic retention, and consequently account for the observed distribution of SORL1-38b transcripts split between soma and dendrites. A similar role for specific exons of BDNF mRNA has previously been demonstrated, showing how individual exons contain motifs responsible for soma retention or dendritic transport [3]. To investigate this, we designed two probes for BaseScope detection of SORL1$38 b$ either including exon 39 (E38bE39) or skipping exon 39 (E38bE40) (Fig. 3b). We then applied the BaseScope Duplex assay for simultaneous visualization of both probes on the same cerebellum sample. In this experiment both probes gave signals within PC dendritic trees (Fig. 3c), indicating that skipping of exon 39 is not related to somatic localization of SORL1-38b transcripts, and suggesting that inclusion of E38b drives SORL1 mRNA into dendrites.

Next, we analysed a number of human brain tissue RNA-seq datasets from available data repositories. By this approach we not only provide further evidence for 

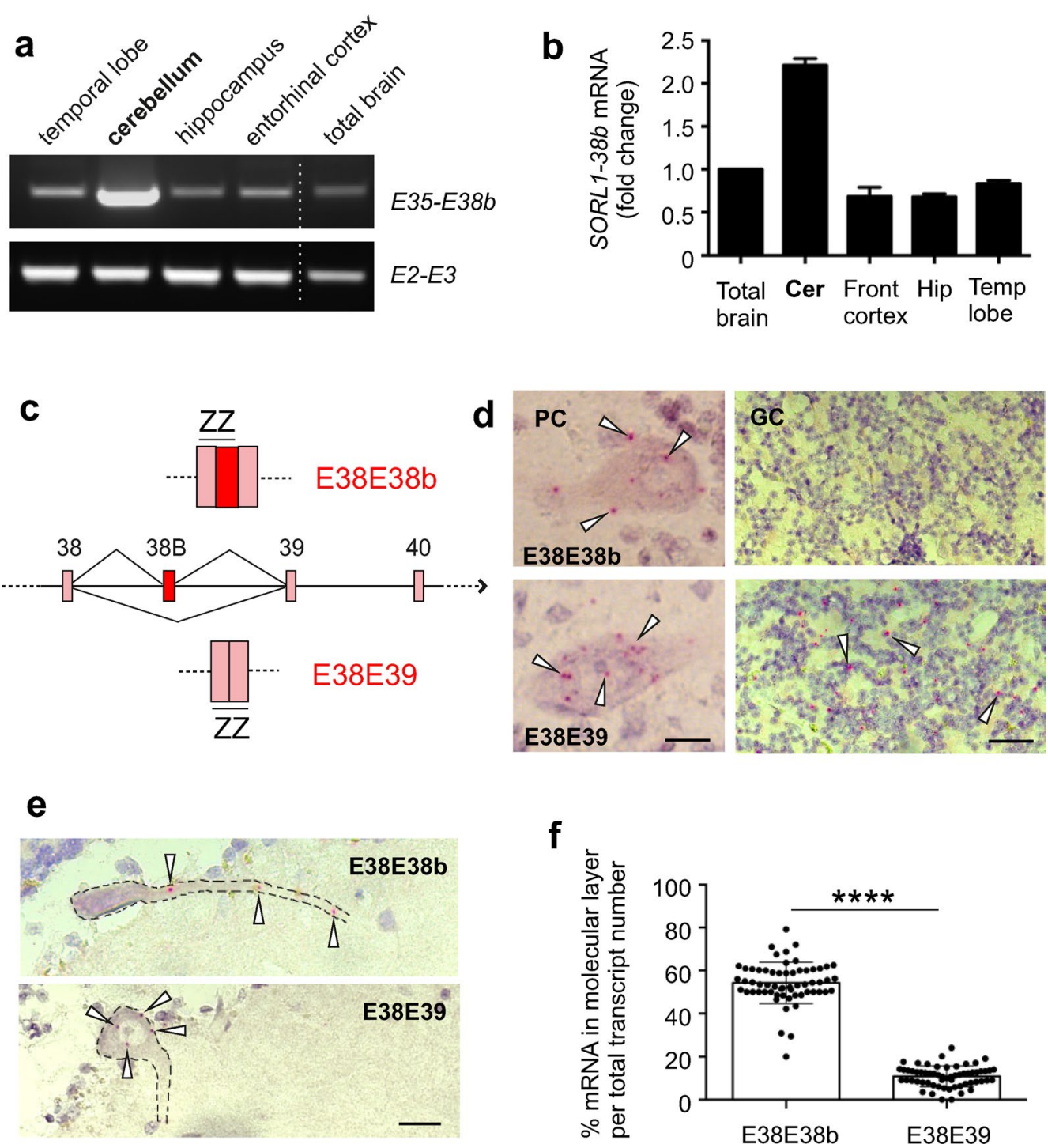

Fig. 2 SORL1-38b is expressed in cerebellar Purkinje cells. a RT-PCR analysis showing expression of SORL1-38b (primers E35-E38b) and control SORL1 transcripts (primers E2-E3) in brain regions. b qPCR analysis of SORL1-38b expression in human brain regions relative to housekeeping genes HPRT1, TFRC and TXNL1. Data are depicted as fold change relative to expression in total brain. Cer cerebellum, Front cortex frontal cortex, Hip hippocampus, Temp lobe temporal lobe. c Schematic representation showing how custom-designed BaseScope double-Z probes target the junctions between exon 38 and 38b (E38E38b), and between exon 38 and 39 (E38E39) to specifically detect E38b-containing transcripts. d BaseScope analysis using E38E38b probe confirms the presence of exon 38b-containing transcripts in human cerebellum. PC Purkinje cells, GC granule cells. Scale bar, $20 \mu m$. e BaseScope images showing that E38E39 probe is mainly localized in the soma of PCs (bottom panel), whereas E38E38b probe signal is found in the soma but also distributed in the dendrites of PCs (top panel). Scale bar, $50 \mu \mathrm{m}$. $\mathbf{f}$ Quantification of the number of SORL 1-38b (E38E38b) and SORL 1-fl (E38E39) transcripts localized in the dendrites of PCs. Individual data points represent quantification from 3 independent brains, and in each section 25 random pictures showing homologous cerebellum regions were used for the analysis ( $n=225$ cells/probe). ${ }^{* * *} p<0.0001$

the presence of SORL1-38b transcripts in all investigated samples, but also confirmed that skipping of exon 39 in the presence of E38b can occur in neurons in various brain regions, including the frontal lobe, hippocampal formations, and superior temporal gyrus (Fig. 3d). In agreement with our experimental findings, the highest number of SORL1-38b transcripts was observed for cerebellum, where it accounts for $\sim 0.6 \%$ of all SORL1 reads.

In situ analyses using probes E38E38b and E38E39 on human entorhinal cortex and hippocampal areas CA4 and dentate gyrus (DG) not only validated the expression of SORL1-38b, but also confirmed how most E38btranscripts are located at the dendritic part of neurons 


\section{(See figure on next page.)}

Fig. 3 Alternative splicing of exon 38b determines soma-dendritic transport of SORL 1 transcripts. a RT-PCR using primers in exon $38 \mathrm{~b}$ and 40 (E38b-E40) revealed the presence of the additional splicing event with skipping of exon 39 downstream the novel 38b cassette. b Schematic of BaseScope probe design for targeting SORL 1-38b transcripts containing (E38bE39, blue) or excluding exon 39 (E38bE40, red). c Representative image of BaseScope Duplex using the probes E38bE39 (blue) and E38bE40 (red) shows that exon 39 is not responsible for soma retention. Scale bar, $20 \mu \mathrm{m}$. d Junction-spanning reads in human brain regions confirm the existence of two SORL 1-38b transcripts from inclusion or exclusion of exon 39. Numbers represent the relative frequency of SORL 1-38b reads among all SORL1 reads (\%). e BaseScope analysis using probes E38E38b and E38E39 shows the presence of extra-somatic SORL 1-38b transcripts in different human brain areas. Signals for E38E39 that represent SORL1-fl are predominantly located in the neuronal soma. Ento ctx entorhinal cortex, Hip hippocampus, DG dentate gyrus. Scale bar, $50 \mu \mathrm{m}$. f Immunostaining of human cerebellum with an antibody raised against the cytoplasmic tail (SORLA-cyt) of SORLA-fl reveals that the full-length protein is mainly located to the soma of PCs. Staining with antibodies against the luminal part of SORLA (SORLA-lum; LR11,5387) shows labeling of soma as well as dendrites of PCs (indicated with arrowheads)

opposed to somatic localization of SORL1-fl (positive for E38E39) in these brain regions (Fig. 3e).

We did several attempts to detect SORLA-38b expression in dendrites of cerebellar sections using our pAb-38b antibody. While we were able to see signal in PC soma and dendrites using this antibody (in line with the presence of SORL1-38b transcripts), we experienced trouble with a background signal in the GCL that confounded the data. We therefore took an alternative approach to support a difference in cellular localization between truncated and SORLA-fl isoforms. Using an antibody that binds the cytoplasmic tail of SORLA, we found that SORLA-fl is almost exclusively located in the PC soma, whereas two different antibodies (IgGs LR11 and 5387), binding to epitopes in the extracellular receptor domain and therefore unable to distinguish between SORLA-fl and SORLA-38b, gave an additional strong signal in the dendritic tree of PCs that was only rarely seen in pictures from immunostainings using the tail antibody (Fig. 3f).

Together, our findings indicate that the SORL1-38b splice variant has a pronounced dendritic localization, likely underlining a yet undefined function. Further studies are necessary to elucidate the molecular drivers as well as the physiological conditions responsible for this dendritic targeting.

\section{The SORL1-38b transcript is downregulated in AD cerebellum}

The cerebellum is historically considered a region of the brain showing little vulnerability for AD [6], although recent studies also link cerebellum to cognitive decline $[16,18]$. Robust SORLA expression in cerebellum in AD has previously been observed $[5,13,30,38]$ despite decreased levels in disease-affected regions including frontal cortex and hippocampus [1, 14, 30]. However, these previous studies did not take into account that alternative isoforms of SORL1 may be altered. As dendritic and synaptic connectivity loss is one of the driving events occurring in AD, we next asked if SORL1-38b levels are affected in the cerebellum of AD patients.
As expected, tissue sections from cerebellum of $\mathrm{AD}$ and non-AD individuals showed no gross abnormalities despite disease status. To ensure comparable RNA quality between $\mathrm{AD}$ and non-AD samples, we hybridized the cerebellar sections with a probe targeting the human PPIB gene, indicating no general RNA decay in these cerebellar AD samples (Additional file 3: Fig. S3). We next performed a quantitative analysis of the expression of SORL1-38b transcripts in PC soma by BaseScope assay by applying probe E38E38b to tissue sections from $3 \mathrm{AD}$ and 3 non-AD individuals (sample data listed in Table 1), and quantified the levels of transcripts. As indication of the expression level, we could group the PCs according to the copy number of E38b-containing transcripts expressed by PCs, and we classified into four groups according to the signal detected per cell soma (Fig. 4a). Surprisingly, we observed a markedly reduced expression of the SORL1-38b isoform in all three AD samples (Fig. 4b).

Detailed quantification based on the signal in the soma revealed that $46.6 \%$ of all measured PCs $(n=1444)$ were negative (group 0) by the E38E38b ISH analysis in non$\mathrm{AD}$ cerebella, and the remaining $53.4 \%$ positive $\mathrm{PCs}$ distributed equally between groups I-II-III (19.7\% group I; $20.5 \%$ group II; $13.2 \%$ group III). However, in cerebella from AD patients, $93.0 \%$ of all PCs showed a lack of SORL1-38b expression $(p<0.001$ compared to non-AD distribution in group 0 ), accompanied by a significant reduction in the number of PCs corresponding to groups I (5.5\%; $p=0.0177)$ and II (1.5\%; $p=0.0002)$ with no cells assigned to group III (Fig. 4b).

In parallel, we tested the same brains to detect SORL1$f l$ transcripts. In line with previous reports that showed robust SORLA expression in AD cerebellum [5, 30], we did also not see any difference for SORL1-fl levels between $\mathrm{AD}$ and non-demented controls (Fig. 4c). Quantification of the hybridization signal for the E38E39 probe showed that the majority of PCs (67.3\%) expresses high levels of SORL1-fl transcript (group III), and that none of the four groups were affected by $\mathrm{AD}$ (group $0, p=0.4197$; 


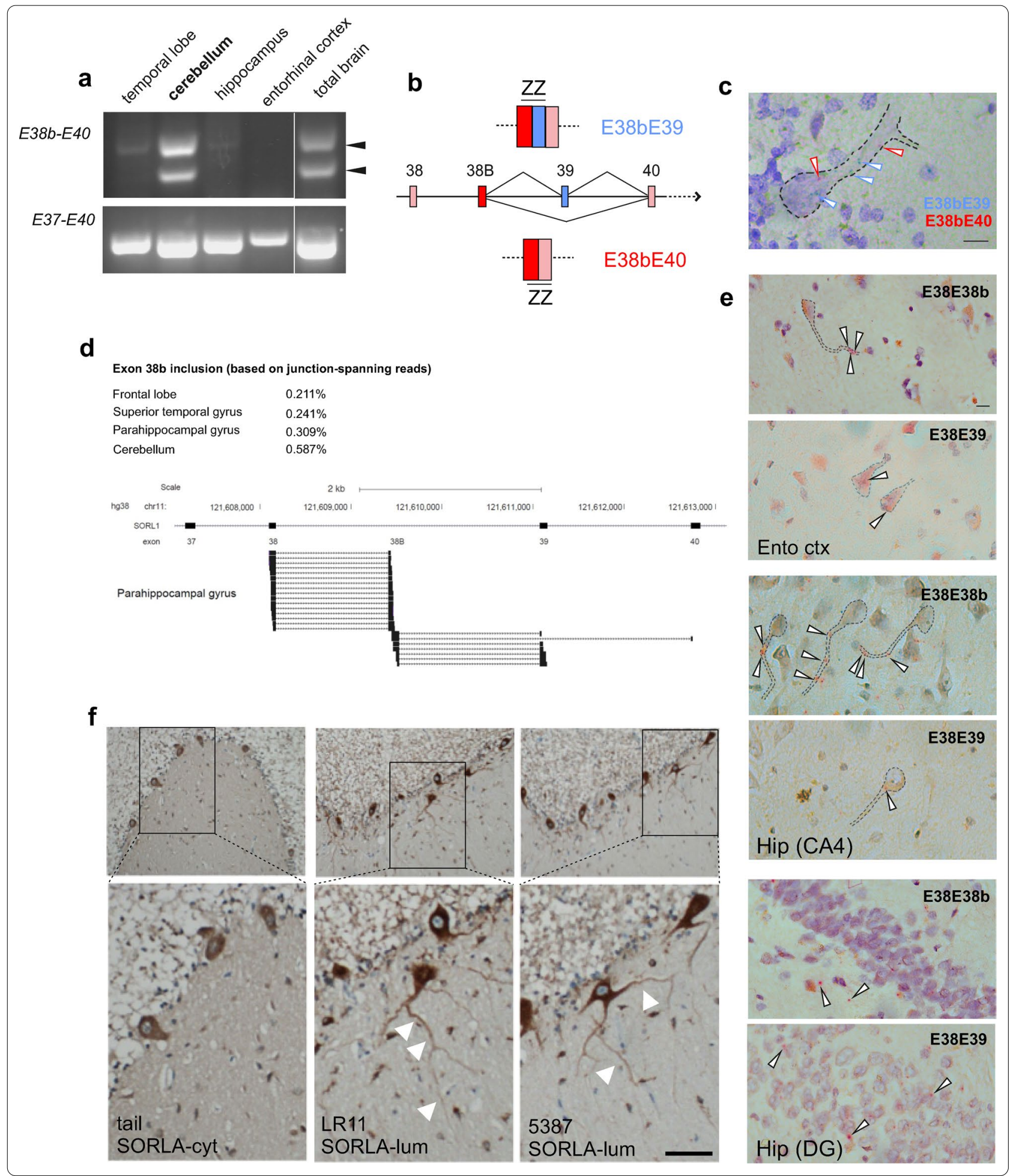



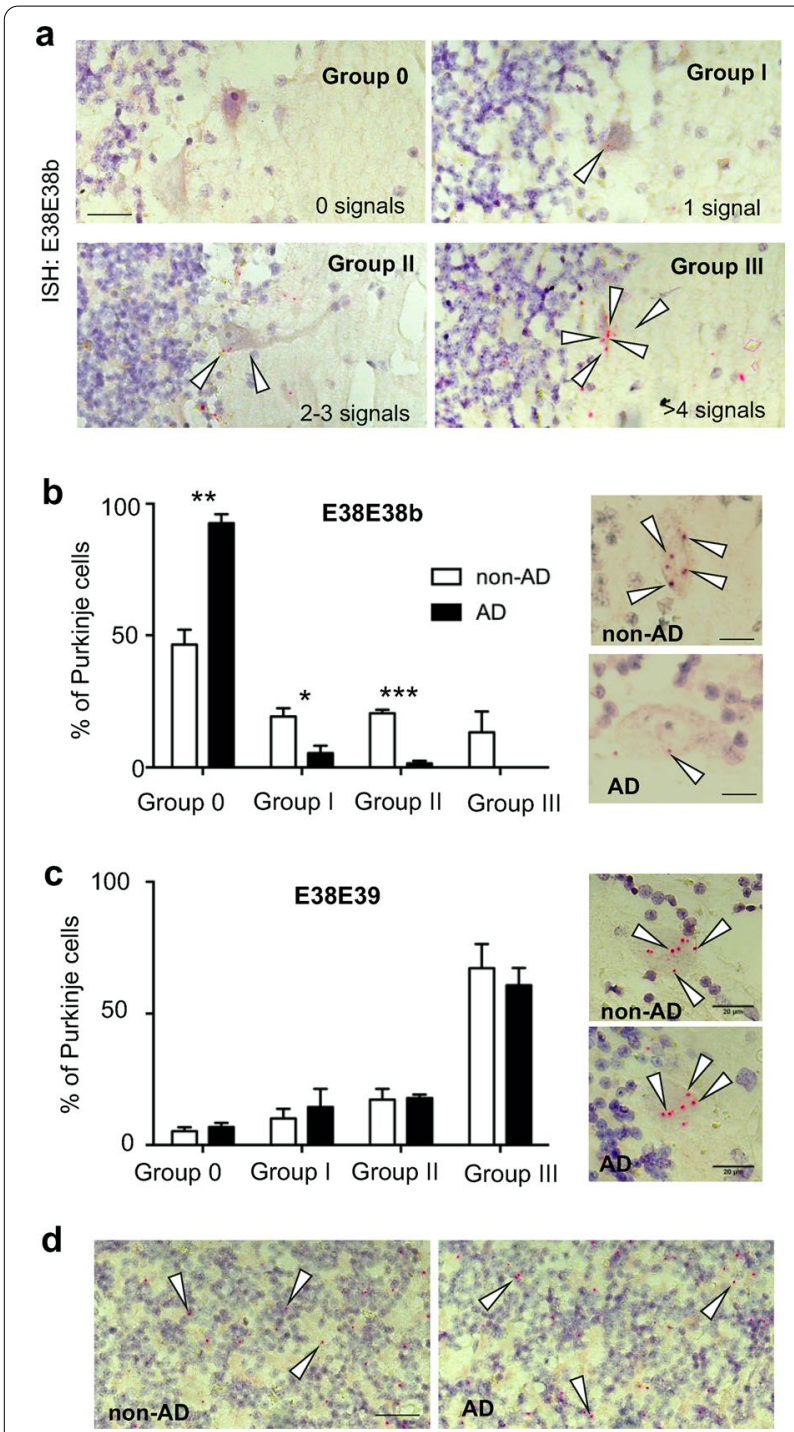

Fig. 4 Reduced expression of the alternatively spliced SORL 1-38b transcript in cerebellum of AD patients. a Representative images of ISH in human cerebella from non-AD individuals using E38E38b probe for detection of SORL 1-38b transcripts. Cells were grouped in 4 different groups according to copy-number of mRNAs: group 0 (0 signals), group I (1 signal), group II (2-3 signals), and group III (>4 signals). Scale bar, 50 m. b Quantification of BaseScope signal in groups of PCs with E38E38b probe confirms significant reduction of $S O R L 1-38 b$ transcripts in the cerebellum from $A D$ patients $(N=3, n=1006$; non-AD; $N=3, n=1444)$. Scale bar, $20 \mu \mathrm{m} .{ }^{*} p<0.05,{ }^{* *} p<0.01,{ }^{* * *} p<0.001$. c Quantification of BaseScope readouts indicates no significant differences of E38E39 probe signal in AD cerebella compared to non-AD controls (non-AD, N=3, n=2936 cells in total; $A D ; N=3, n=2768$ cells in total). Scale bar, $20 \mu \mathrm{m}$. $\mathbf{d}$ Representative images of ISH signal using E38E39 probe show that SORL 1-fl is similarly expressed in GCs in non-AD and AD cerebella. Scale bar, $50 \mu \mathrm{m}$

group I, $p=0.4674$; group II, $p=0.8955$; group III, $p=0.5474$ ) (Fig. 4c). Similarly, we did not observe any decrease in the signal for SORL1-fl in the GCL (Fig. $4 \mathrm{~d}$ ).
These findings confirmed SORL1- $f l$ is abundantly and robustly expressed in the human cerebellum, and its expression is not affected by $\mathrm{AD}$ in this brain region.

\section{Replication analysis of transcript levels}

To replicate the finding of lowered SORL1-38b expression in brains from AD patients, we used Taqman qPCR to quantify $S O R L 1-38 b$ levels in AD versus non-AD brains in two additional independent cohorts. First, we investigated $25 \mathrm{AD}$ and 25 non-AD post-mortem cerebella samples identifying a mean 2.0-fold down-regulation of SORL1-38b transcript levels in AD cerebellum compared to controls $(p=0.0010)$ (Fig. 5a). Of notice, this cohort was previously analyzed and showed no reduction of SORL1- $f l$ in AD patients compared to controls [5], demonstrating again that the two SORL1 isoforms are differently affected by AD. Consistent with this, a qPCR analysis of $14 \mathrm{AD}$ and 6 non-AD cerebella samples identified a mean 2.4-fold down-regulation of SORL1-38b transcripts in AD brains compared to non-AD brains $(p=0.076)$ and no change in SORL1- $f$ (Fig. 5b). Quantification of the PC marker Calbindin relative to the reference genes showed no differences between $\mathrm{AD}$ and non-AD samples from the UW cohort $(\Delta \Delta \mathrm{Ct}=-0.08$; $p=0.869$ ). A meta analysis of the two cohorts (in total 39 AD; 31 non-AD) found a mean 2.1-fold decrease of SORL1-38b in AD ( $\mathrm{p}_{\text {meta }}=0.00035$ ) (Fig. 5c).

\section{Transcript levels stratified by SNP24}

The first association study focusing on SORL1, carried out by Rogaeva and coworkers in 2007 [36], identified an association with $\mathrm{AD}$ for two different haplotypes in $S O R L 1$, more specifically a $5^{\prime}$ risk-haplotype and a $3^{\prime}$ riskhaplotype in the gene [36]. While the association with $5^{\prime}$ risk-haplotype has been linked to reduced BDNF-induced SORL1 activity [49], the underlying reason for the 3 'risk haplotype association remains unclear. In 2019, a large GWAS of AD identified a single risk locus in SORL1 with lead SNP rs11218343 [20]. The locus spans around $68 \mathrm{~kb}$ (from rs7946599 to rs75439772) and includes E38b as well as the $3^{\prime}$ haplotype [20] (Additional file 4: Fig. S4a). One of the SNPs tagging the $3^{\prime}$ haplotype is rs2282649, labelled SNP24 in the Rogaeva study [36]. Because of the association previously reported and the genomic position of SNP24 close to E38b (around $1200 \mathrm{bp}$ ), we speculated if rs2282649/SNP24 was associated with AD, and if SNP24 genotypes correlated with SORL1-38b levels. Out of the 50 Mayo cerebellum samples, 35 samples had genotypes available for rs2282649/SNP24 from wholegenome sequencing in the AMP-AD knowledge portal. The samples from UW were genotyped for SNP24 and selected to enrich for individuals with the T/T genotype. 


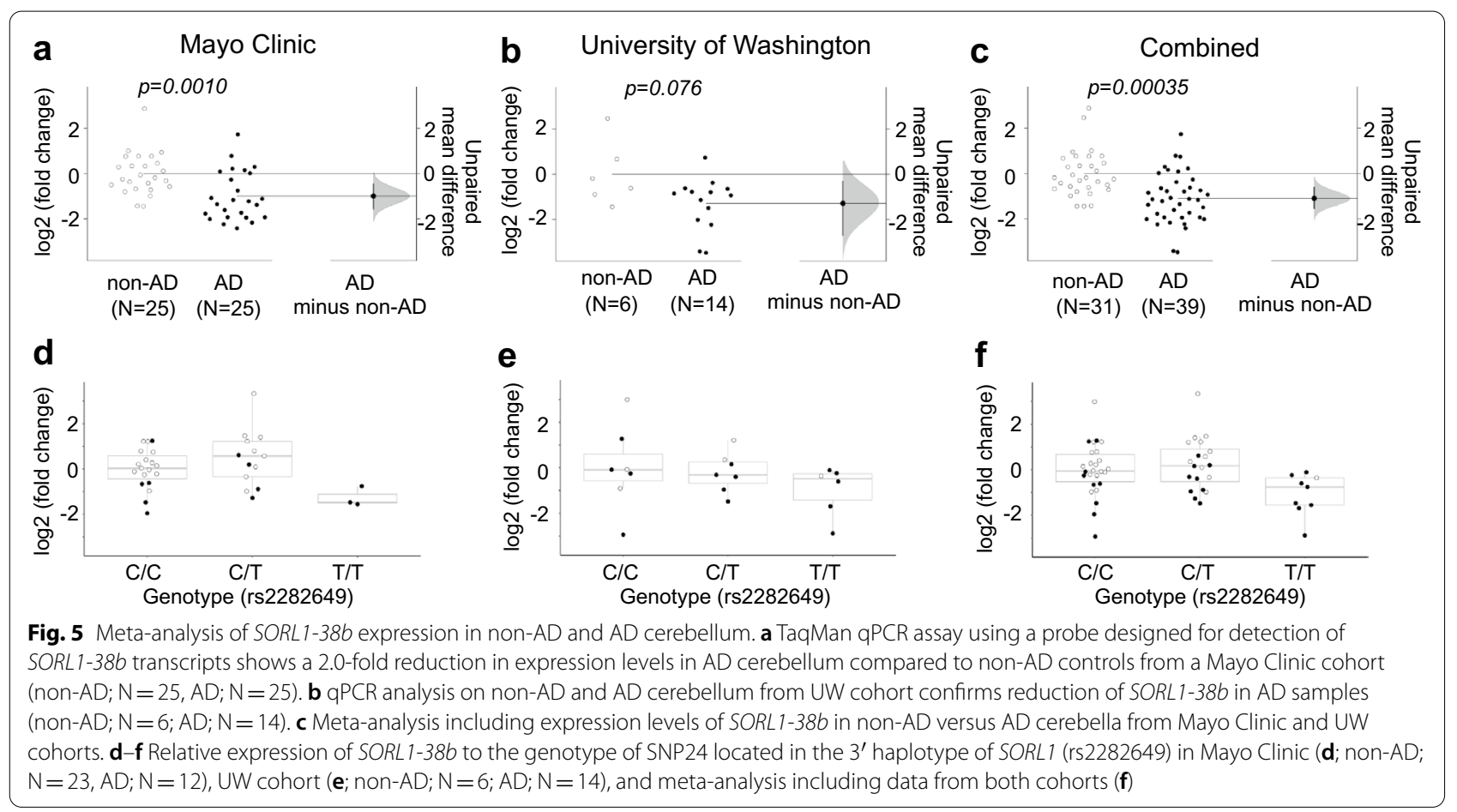

In the Mayo subset (12 AD and 23 non-AD) the SNP24 allele frequency of the risk allele (T-allele) was higher among AD samples $(41.7 \%)$ compared to non-AD samples $(19.6 \%)(p=0.09)$, supporting a trend for association of this variant with AD in this small sample. Because samples from the UW cohort were selected for qPCR based on their SNP24 genotype, allele frequencies are not representative for $\mathrm{AD}$ cases and controls, and they were not included in the association analysis with disease status.

Stratifying the Mayo and the UW samples according to SNP24 genotypes revealed a decreasing tendency in SORL1-38b levels in carriers of the risk genotype T/T of SNP24 (rs2282649) (Fig. 5d, e) $\left(p_{\text {meta }}=0.14\right.$, analysis adjusted for AD status) (Fig. 5f). To determine if SNP24 is a true eQTL for SORL1-38b levels, a larger number of samples is needed, with both genotypes and E38b transcript levels available.

To test if any stronger eQTLs for $38 \mathrm{~b}$ existed in the SORL1 locus, genetic variants in SORL1 as well as upand downstream were downloaded from the AMP-AD knowledge portal for the 35 Mayo samples. Apart from a small cluster of SNPs in the SORL1 promoter, which was to be expected, no other strong eQTL could be identified for E38b-transcript levels within the SORL1 locus (Additional file 4: Fig. S4b). Future studies with a larger number of individuals, including also carriers of the GWAS index SNPs, could reveal if the mechanism underlying common-variant association of SORL1 with AD involves the E38b transcript.

\section{Discussion}

We have previously shown how a SORL1 transcript that differs from full-length is generated by an alternative transcriptional start site [5], but SORL1-38b is to our knowledge the first alternatively spliced isoform of the AD gene SORL1 that has been documented. Interestingly, our findings showed that the inclusion of E38b in transcripts primes mRNA translocation targeting SORL1-38b to extra-somatic compartments in neurons. Such asymmetrical distribution of mRNA may be more thermodynamically efficient than transporting proteins as fewer mRNA molecules need to be mobilized [8]. Neurons in particular rely on mRNA translocation as a mechanism to coordinate regional and temporal changes in protein levels in both axonal and dendritic compartments, as well as by local protein synthesis to affect synaptic plasticity of individual synapses [7].

For our studies, we focused on the cerebellum showing the highest relative expression of SORL1-38b, like for SORL1-fl. Also, we confirmed both the expression of this isoform and its dendritic localization in neurons of hippocampus and cortical regions that are affected by AD. This suggests the cerebellum as a model system to study SORL1-38b biology, allowing the translation of our findings to other brain areas affected by AD pathology. The high cerebellar expression may reflect the fact that 
Purkinje neurons have the most elaborate dendritic trees among neurons in the brain [42], with a huge number of dendritic spines that could be particularly dependent on $S O R L 1$ splicing. The role of SORL1-38b is not clear, but it may act similarly to protocadherins where AS has been reported to influence self-avoidance of dendrites in cerebellar PC [23]. Accordingly, SORL1-38b could play an important role to provide synapse and neuronal identity.

Here, we provide evidence that $S O R L 1-38 b$ is decreased in the cerebellum of patients with AD, using samples from three independent cohorts. While SORL1$f l$ is not decreased in cerebellum of AD patients $[2,5$, 30 ], we found a significant decrease of SORL1-38b in AD patients from all three cohorts. This is interesting as cerebellum, which is usually described as unaffected in AD, recently has gained attention as playing a role in cognitive decline [18], with evidence showing that networkbased degeneration in AD extends to the cerebellum [16]. Although AS has previously been studied in relation to AD [21, 37], this is the first description of a SORL1 isoform affected in the cerebellum of AD patients.

The specific decrease of SORL1-38b levels in AD suggested a unique difference in either production or degradation between this isoform and SORL1-fl. An ADdependent change of the splicing machinery necessary for E38b inclusion may explain differences in the production of the two isoforms. Alternatively, a decrease in the dendritic machinery responsible for transporting the mRNA or being related to previously reported synaptic and dendritic loss in AD including loss of synapses of PCs in $\mathrm{AD}[25,26]$ could explain the observed decrease. However, any mechanism where SORL1-38b loss is a consequence of its localization into dendrites/synapses would seem to require a feedback mechanism in order to additionally explain the observed decrease of SORL1-38b in the neuronal soma by ISH. Interestingly, the most severe reduction of SORL1-38b in AD cerebella was found in the NBB cohort, only including samples at advanced stages compared to UW and Mayo cohorts. This could reflect a positive correlation between severity of E38b-transcripts downregulation and stage of AD.

If the identified reduction of $\mathrm{E} 38 \mathrm{~b}$ is a consequence of synaptic loss due to AD, our findings may also be relevant for other neurodegenerative disorders like Parkinson's disease and frontotemporal dementia. It would therefore be of interest for future studies to determine SORL1-38b levels also for non-AD degenerative diseases, as well as looking further into the possible association between SORL1-38b and SNP24 that is specifically reported to associate with $\mathrm{AD}[36]$.

Translation of transcripts including E38b leads to production of a truncated SORLA isoform lacking four 3Fn-domains as well as transmembrane and cytoplasmic domains that are all present in SORLA-fl. This suggests different functions of the two isoforms, in line with multiple studies reporting that locally synthesized proteins are most often structurally and functionally distinct from proteins present at the same cellular site but being transported to their destination [8]. Usually, transcripts containing a premature stop codon (PSC) are targets of the nonsense mediated decay (NMD) pathway. However, it has been previously shown that a human specific splice variant of the Survival Motor Neuron 1 (SMN1) gene holding a PSC, after introduction of a novel exon, is able to escape NMD and be locally translated in neuronal terminals [40]. Similarly, NMD degradation of SORL1$38 \mathrm{~b}$ may be prevented by trafficking the transcript to dendrites where the truncated SORLA-38b protein is produced.

SORLA-fl is known to regulate sorting of a number of cargo proteins including APP and the Amyloid- $\beta$ peptide $[1,9]$, in a process that requires complex formation between the SORLA tail domain and cytosolic trafficking factors, most notably the retromer complex [15]. This activity determines sorting of SORLA and its cargo in Golgi and endosomal compartments of the neuronal soma. Here, we were puzzled why SORLA-38b is not merely being secreted in the absence of the transmembrane anchor, as a construct encoding the longer extracellular region of SORLA containing all six 3Fn-domains become efficiently secreted [39]. Our experiment to determine if the presence of the E38b-encoding 22 amino acids was responsible for this cellular retention showed that the E38b-encoded peptide was not responsible for the lack of secretion. Subsequent studies revealed that the ability to leave the cell is determined by the presence of the fifth 3Fn-domain in the SORLA ectodomain, likely reflecting conformational rearrangements of the SORLA extracellular part in the absence of this domain. This strongly support our hypothesis that SORLA-38b has its own cellular function unrelated to SORLA-fl.

The exact function of the shorter SORLA-38b isoform is not clear, but it is very tempting to speculate a role related to synaptic plasticity, which is the most frequent function associated with localized protein synthesis in dendrites [45]. The study of the function of SORLA$38 \mathrm{~b}$ is complicated as it is not expressed in rodents. We attempted to differentiate neurons from human induced pluripotent stem cells, however the applied protocol previously shown to provide a model for studying the function of SORLA-fl [22] unfortunately failed to induce sufficient levels of SORL1-38b expression (determined by qPCR; data not shown). This observation is in line with our present findings where only a subset of neurons expresses the alternatively spliced isoform. We suggest future studies should apply protocols for differentiation 
into PCs $[47,48]$ to increase the possibility that the induced neurons will express sufficient SORLA-38b to allow for functional studies.

SORLA has previously been speculated to act as an adhesion protein, because 3Fn-domains are often present in adhesion molecules, including protocadherins, immunoglobulin superfamily and contactins $[4,10]$. Interestingly, inclusion of an alternative exon in CNTN4 (encoding Contactin-4) introduces a novel stop codon and generates a truncated protein with deletion of several 3Fn-domains [50] similarly to what we here describe for SORLA-38b. Detection of the truncated form of Contactin-4 in human brain samples confirmed the production of a stable isoform. AS of proteins involved in cellular adhesion has been reported to generate extensive diversity for neuronal intercellular recognition, often in a cell type specific way. Such splice-dependent interactions play fundamental roles in neural circuit establishment and maintenance, including repulsion and attraction allowing neurites to form specific synapses [34]. Local synthesis of SORLA-38b in dendrites would be in perfect agreement with such a function in cellular contact formation at the synapse, and may also relate to our observation that expression of SORLA-38b is restricted to a subset of neurons-providing neuronal identity-in comparison to SORLA-fl with a broader expression profile and function.

In conclusion, for the characterization of the lowly expressed SORL1-38b we have established several methods including the development of a specific antibody, the application of E38b-specific probes for ISH and the optimization of qPCR assays. Altogether, these made the identification of an otherwise hardly detectable splice isoform possible, providing valuable information about SORL1 transcriptomic profile.

Although the study revealed novel findings about SORL1, a few limitations need to be mentioned. First, the low expression level of SORL1-38b challenged the detection of this transcript in other brain regions than the cerebellum. Thus, the implementation of more sensitive techniques, as well as the optimization of established protocols, will aim at confirming our data in non-cerebellar areas. Second, the small sample size of the cerebellum cohorts limited the analysis of SORL1-38b expression in relation to the risk T/T (SNP24) genotype. Future studies including larger cohorts will help clarifying how SORL1$38 b$ associates with SNP24.

\section{Conclusions}

Here, we provide the first detailed characterization of an alternatively spliced transcript of the SORL1 gene. Our results highlight two major novel findings in SORL1 research: the dendritic localization of SORL1-38b transcripts and the specific reduction of E38b levels in cerebellum from AD patients despite SORL1- $f$ transcripts being unaltered. Altogether, these data add another layer of complexity to the understanding of SORL1 biology.

\section{Supplementary Information}

The online version contains supplementary material available at https://doi. org/10.1186/s40478-021-01140-7.

Additional file 1. Figure S1: SORLA-38b is retained intracellularly in HEK cells. a Substitutions of cysteine to alanine at position 1 and 13 of the unique tail encoded by E38b do not alter the secretion of SORLA-38b in the medium. Non transf non transfected cells. $\mathbf{b}$ WB analysis of lysates and media from $\mathrm{CHO}$ cells transiently transfected with SORLA-fl, SORLA variants where stop mutations were introduced at the boundary following each 3Fn domain, or a SORLA variant lacking the cytoplasmic tail (SORLA$\triangle C D)$. Notably, constructs lacking the $3 \mathrm{Fn} 5$ are most strongly retained intracellularly (indicated with arrowheads).

Additional file 2. Figure S2: Analysis of the $3^{\prime}$ end of SORL 1-38b. Agarose gel showing 3' RACE products for SORL 1-fl and SORL 1-38b. Bands indicated with arrows have been sequenced.

Additional file 3. Figure S3: Control of RNA quality in non-AD and AD cerebella. Samples hybridization with PPIB probe showed no distinct difference in the signal from non-AD compared to AD cerebella.

Additional file 4. Figure S4: Analysis of genetic variants affecting the expression of E38b. a Representation of the boundaries of the $3^{\prime}$ end locus in SORL 1 including localization of E38b. $\mathbf{b}$ eQTL mapping of SORL 1 gene did not show significant association with E38b expression (SNP24: rs2282649).

\section{Acknowledgements}

We are grateful to Sandra Bonnesen, Anja Aagaard, Benedicte Vestergaard, Mette Singers, and Anne Kerstine Glintborg Jensen for technical assistance. We thank NBB, Mayo Clinic and UW for providing human samples necessary to carry out this project.

\section{Authors' contributions}

GM, MK, AMGJ, JR, RC, BEZ, CV, MMJ, NW conducted laboratory experiments. MK supervised the generation of the polyclonal antibody against SORLA-38b. IEH supervised histological analyses. MA, SL conducted experiments and analyses for the Mayo Clinic samples. NET provided oversight, resources for the Mayo Clinic studies. PLM performed the statistical analyses in R including the eQTL analysis. PNV conducted RNA-seq read alignment on human brain regions. JEY provided oversight and resources for studies conducted at University of Washington. IEH, CBV, and AN provided expert advice and assisted with data interpretation. GM, MN, and OA drafted the manuscript. All authors read and approved the final manuscript.

\section{Funding}

Study data were provided by the following sources: The Mayo Clinic Alzheimers Disease Genetic Studies, led by Dr. Nilüfer Ertekin-Taner and Dr. Steven G. Younkin, Mayo Clinic, Jacksonville, FL using samples from the Mayo Clinic Study of Aging, the Mayo Clinic Alzheimers Disease Research Center, and the Mayo Clinic Brain Bank. Data collection was supported through funding by NIA Grants P50 AG016574, R01 AG032990, U01 AG046139, R01 AG018023, U01 AG006576, U01 AG006786, R01 AG025711, R01 AG017216, R01 AG003949, NINDS Grant R01 NS080820, CurePSP Foundation, and support from Mayo Foundation. Study data includes samples collected through the Sun Health Research Institute Brain and Body Donation Program of Sun City, Arizona. The Brain and Body Donation Program is supported by the National Institute of Neurological Disorders and Stroke (U24 NS072026 National Brain and Tissue 
Resource for Parkinsons Disease and Related Disorders), the National Institute on Aging (P30 AG19610 Arizona Alzheimers Disease Core Center), the Arizona Department of Health Services (contract 211002, Arizona Alzheimers Research Center), the Arizona Biomedical Research Commission (contracts 4001, 0011 , 05-901 and 1001 to the Arizona Parkinson's Disease Consortium) and the Michael J. Fox Foundation for Parkinsons Research. Autopsy materials used in this study were in part obtained from the University of Washington Neuropathology Core, which is supported by the Alzheimer's Disease Research Center (AG05136), the Adult Changes in Thought Study (AG006781), the Pacific Udall Center 3P50NS062684, and DOD HU00011920008. Research in OA lab was supported by the Danish Alzheimer's research foundation, the Mærsk-Møller Foundation, the Jascha Foundation, and Aarhus University Graduate School Health. Research conducted in AN lab was supported by PROMEMO-Center for Proteins in Memory, a Center of Excellence funded by the Danish National Research Foundation (Grant DNRF133).

\section{Availability of data and materials}

The results published here are in part based on data obtained from the AMP-AD Knowledge Portal (https://adknowledgeportal.synapse.org/). Whole genome-sequence data for 35 of the Mayo Clinic samples is available via the AD Knowledge Portal (https://adknowledgeportal.synapse.org, https://doi. org/10.7303/syn2580853).

\section{Declarations}

\section{Ethics approval and consent to participate}

The use of human brains from non-AD and $A D$ individuals was approved by international review boards for Mayo, UW and NBB cohorts.

\section{Consent for publication}

Not applicable.

\section{Competing interests}

The authors declare no competing interests.

\section{Author details}

${ }^{1}$ Department of Biomedicine, Aarhus University, Høegh-Guldbergs Gade 10, 8000 Aarhus C, Denmark. ${ }^{2}$ Department of Neuroscience, Mayo Clinic, Jacksonville, FL 32224, USA. ${ }^{3}$ Department of Biochemistry, Jena University Hospital, Jena, Germany. ${ }^{4}$ Department of Clinical Medicine, Aarhus University, Aarhus C, Denmark. ${ }^{5}$ Department of Pathology, Randers Regional Hospital, Randers, Denmark. ${ }^{6}$ Department of Medicine, Division of Medical Genetics, University of Washington, Seattle, WA, USA. ${ }^{7}$ Center for Proteins in Memory - PROMEMO, Danish National Research Foundation, Department of Biomedicine, Aarhus University, Aarhus C, Denmark. ${ }^{8}$ Department of Neurology, Mayo Clinic, Jacksonville, FL 32224, USA. ${ }^{9}$ Department of Pathology, Institute for Stem Cell and Regenerative Medicine, University of Washington, Seattle, WA, USA.

Received: 22 February 2021 Accepted: 26 February 2021 Published online: 16 March 2021

\section{References}

1. Andersen OM, Reiche J, Schmidt V, Gotthardt M, Spoelgen R, Behlke J, von Arnim CAF, Breiderhoff T, Jansen P, Wu X, Bales KR, Cappai R, Masters CL, Gliemann J, Mufson EJ, Hyman BT, Paul SM, Nykjaer A, Willnow TE (2005) Neuronal sorting protein-related receptor sorLA/LR1 1 regulates processing of the amyloid precursor protein. Proc Natl Acad Sci USA 102:13461-13466. https://doi.org/10.1073/pnas.0503689102

2. Andersen OM, Rudolph I-M, Willnow TE (2016) Risk factor SORL 1: from genetic association to functional validation in Alzheimer's disease. Acta Neuropathol 132:653-665. https://doi.org/10.1007/s00401-016-1615-4

3. Baj G, Leone E, Chao MV, Tongiorgi E (2011) Spatial segregation of BDNF transcripts enables BDNF to differentially shape distinct dendritic compartments. Proc Natl Acad Sci USA 108:16813-16818. https://doi.org/10. 1073/pnas.1014168108

4. Bencharit S, Bin CC, Siddiqui A, Howard-Williams EL, Sondek J, ZuobiHasona K, Aukhil I (2007) Structural insights into fibronectin type III domain-mediated signaling. J Mol Biol 367:303-309. https://doi.org/10. 1016/j.jmb.2006.10.017

5. Blechingberg J, Poulsen ASA, Kjolby M, Monti G, Allen M, Ivarsen AK Lincoln SJ, Thotakura G, Vaegter CB, Ertekin-Taner N, Nykjaer A, Andersen OM (2018) An alternative transcript of the Alzheimer's disease risk gene SORL1 encodes a truncated receptor. Neurobiol Aging 71:266.e11-266. e24. https://doi.org/10.1016/j.neurobiolaging.2018.06.021

6. Braak H, Braak E (1991) Neuropathological stageing of Alzheimer-related changes. Acta Neuropathol 82:239-259. https://doi.org/10.1007/bf003 08809

7. Bramham CR, Wells DG (2007) Dendritic mRNA: transport, translation and function. Nat Rev Neurosci 8:776-789. https://doi.org/10.1038/nrn2150

8. Buxbaum AR, Yoon YJ, Singer RH, Park HY (2015) Single-molecule insights into mRNA dynamics in neurons. Trends Cell Biol 25:468-475. https://doi. org/10.1016/j.tcb.2015.05.005

9. Caglayan S, Takagi-Niidome S, Liao F, Carlo A-S, Schmidt V, Burgert T, Kitago Y, Fuchtbauer E-M, Fuchtbauer A, Holtzman DM, Takagi J, Willnow TE (2014) Lysosomal sorting of amyloid-beta by the SORLA receptor is impaired by a familial Alzheimer's disease mutation. Sci Transl Med 6:223ra20. https://doi.org/10.1126/scitranslmed.3007747

10. Campbell ID, Spitzfaden C (1994) Building proteins with fibronectin type III modules. Structure 2:333-337. https://doi.org/10.1016/s0969-2126(00) 00034-4

11. Campion D, Charbonnier C, Nicolas G (2019) SORL1 genetic variants and Alzheimer disease risk: a literature review and meta-analysis of sequencing data. Acta Neuropathol 138:173-186. https://doi.org/10.1007/ s00401-019-01991-4

12. Ciolli Mattioli C, Rom A, Franke V, Imami K, Arrey G, Terne M, Woehler A, Akalin A, Ulitsky I, Chekulaeva M (2019) Alternative 3' UTRs direct localization of functionally diverse protein isoforms in neuronal compartments. Nucl Acids Res 47:2560-2573. https://doi.org/10.1093/nar/gky1270

13. Dodson SE, Andersen OM, Karmali V, Fritz JJ, Cheng D, Peng J, Levey Al, Willnow TE, Lah JJ (2008) Loss of LR11/SORLA enhances early pathology in a mouse model of amyloidosis: evidence for a proximal role in Alzheimer's disease. J Neurosci 28:12877-12886. https://doi.org/10.1523/JNEUR OSCI.4582-08.2008

14. Dodson SE, Gearing M, Lippa CF, Montine TJ, Levey Al, Lah JJ (2006) LR11/ SorLA expression is reduced in sporadic Alzheimer disease but not in familial Alzheimer disease. J Neuropathol Exp Neurol 65:866-872. https:// doi.org/10.1097/01.jnen.0000228205.19915.20

15. Fjorback AW, Seaman M, Gustafsen C, Mehmedbasic A, Gokool S, Wu C, Militz D, Schmidt V, Madsen P, Nyengaard JR, Willnow TE, Christensen EI, Mobley WB, Nykjaer A, Andersen OM (2012) Retromer binds the FANSHY sorting motif in SorLA to regulate amyloid precursor protein sorting and processing. J Neurosci 32:1467-1480. https://doi.org/10.1523/JNEUROSCI. 2272-11.2012

16. Guo CC, Tan R, Hodges JR, Hu X, Sami S, Hornberger M (2016) Networkselective vulnerability of the human cerebellum to Alzheimer's disease and frontotemporal dementia. Brain 139:1527-1538. https://doi.org/10. 1093/brain/aww003

17. Holstege $H$, van der Lee $S J$, Hulsman $M$, Wong TH, van Rooij JG, Weiss $M$, Louwersheimer E, Wolters FJ, Amin N, Uitterlinden AG, Hofman A, Ikram MA, van Swieten JC, Meijers-Heijboer H, van der Flier WM, Reinders MJ, van Duijn CM, Scheltens P (2017) Characterization of pathogenic SORL1 genetic variants for association with Alzheimer's disease: a clinical interpretation strategy. Eur J Hum Genet 25:973-981. https://doi.org/10.1038/ ejhg.2017.87

18. Jacobs HIL, Hopkins DA, Mayrhofer HC, Bruner E, van Leeuwen FW, Raaijmakers W, Schmahmann JD (2018) The cerebellum in Alzheimer's disease: evaluating its role in cognitive decline. Brain 141:37-47. https://doi.org/ 10.1093/brain/awx194

19. Jacobsen L, Madsen P, Moestrup SK, Lund AH, Tommerup N, Nykjaer A, Sottrup-Jensen L, Gliemann J, Petersen CM (1996) Molecular characterization of a novel human hybrid-type receptor that binds the alpha2-macroglobulin receptor-associated protein. J Biol Chem 271:31379-31383

20. Jansen IE, Savage JE, Watanabe K, Bryois J, Williams DM, Steinberg S, Sealock J, Karlsson IK, Hagg S, Athanasiu L, Voyle N, Proitsi P, Witoelar A, Stringer S, Aarsland D, Almdahl IS, Andersen F, Bergh S, Bettella F, Bjornsson S, Braekhus A, Brathen G, de Leeuw C, Desikan RS, Djurovic S, Dumitrescu L, Fladby T, Hohman TJ, Jonsson PV, Kiddle SJ, Rongve A, Saltvedt I, Sando SB, Selbaek G, Shoai M, Skene NG, Snaedal J, Stordal E, 
Ulstein ID, Wang Y, White LR, Hardy J, Hjerling-Leffler J, Sullivan PF, van der Flier WM, Dobson R, Davis LK, Stefansson H, Stefansson K, Pedersen NL, Ripke S, Andreassen OA, Posthuma D (2019) Genome-wide meta-analysis identifies new loci and functional pathways influencing Alzheimer's disease risk. Nat Genet 51:404-413. https://doi.org/10.1038/ s41588-018-0311-9

21. De Jonghe C, Cruts M, Rogaeva EA, Tysoe C, Singleton A, Vanderstichele $\mathrm{H}$, Meschino W, Dermaut B, Vanderhoeven I, Backhovens H, Vanmechelen E, Morris CM, Hardy J, Rubinsztein DC, St George-Hyslop PH, Van Broeckhoven C (1999) Aberrant splicing in the presenilin-1 intron 4 mutation causes presenile Alzheimer's disease by increased Abeta42 secretion. Hum Mol Genet 8:1529-1540. https://doi.org/10.1093/hmg/8.8.1529

22. Knupp A, Mishra S, Martinez R, Braggin JE, Szabo M, Hailey DW, Small SA Jayadev S, Young JE (2020) Depletion of the AD risk gene SORL1 selectively impairs neuronal endosomal traffic independent of amyloidogenic APP processing. Cell Rep 31(9):107719. https://doi.org/10.1101/2020.01. 06.896670

23. Lefebvre JL, Kostadinov D, Chen WV, Maniatis T, Sanes JR (2012) Protocadherins mediate dendritic self-avoidance in the mammalian nervous system. Nature 488:517-521. https://doi.org/10.1038/nature11305

24. Maciel R, Bis DM, Rebelo AP, Saghira C, Zuchner S, Saporta MA (2018) The human motor neuron axonal transcriptome is enriched for transcripts related to mitochondrial function and microtubule-based axonal transport. Exp Neurol 307:155-163. https://doi.org/10.1016/j.expneurol.2018. 06.008

25. Mavroudis IA, Fotiou DF, Adipepe LF, Manani MG, Njau SD, Psaroulis D, Costa VG, Baloyannis SJ (2010) Morphological changes of the human purkinje cells and deposition of neuritic plaques and neurofibrillary tangles on the cerebellar cortex of Alzheimer's disease. Am J Alzheimers Dis Other Demen 25:585-591. https://doi.org/10.1177/1533317510382892

26. Mavroudis IA, Manani MG, Petrides F, Petsoglou K, Njau SD, Costa VG, Baloyannis SJ (2013) Dendritic and spinal pathology of the Purkinje cells from the human cerebellar vermis in Alzheimer's disease. Psychiatr Danub 25:221-226

27. McKhann G, Drachman D, Folstein M, Katzman R, Price D, Stadlan EM (1984) Clinical diagnosis of Alzheimer's disease: report of the NINCDSADRDA Work Group under the auspices of Department of Health and Human Services Task Force on Alzheimer's Disease. Neurology 34:939-944. https://doi.org/10.1212/wnl.34.7.939

28. Motoi Y, Aizawa T, Haga S, Nakamura S, Namba Y, Ikeda K (1999) Neuronal localization of a novel mosaic apolipoprotein E receptor, LR11, in rat and human brain. Brain Res 833:209-215

29. Nicolas G, Charbonnier C, Wallon D, Quenez O, Bellenguez C, GrenierBoley B, Rousseau S, Richard A-C, Rovelet-Lecrux A, Le Guennec K, Bacq D, Garnier J-G, Olaso R, Boland A, Meyer V, Deleuze J-F, Amouyel P, Munter HM, Bourque G, Lathrop M, Frebourg T, Redon R, Letenneur L, Dartigues J-F, Genin E, Lambert J-C, Hannequin D, Campion D (2016) SORL1 rare variants: a major risk factor for familial early-onset Alzheimer's disease. Mol Psychiatry 21:831-836. https://doi.org/10.1038/mp.2015.121

30. Offe K, Dodson SE, Shoemaker JT, Fritz JJ, Gearing M, Levey Al, Lah JJ (2006) The lipoprotein receptor LR11 regulates amyloid beta production and amyloid precursor protein traffic in endosomal compartments. J Neurosci 26:1596-1603. https://doi.org/10.1523/JNEUROSCI.4946-05. 2006

31. Pan Q, Shai O, Lee LJ, Frey BJ, Blencowe BJ (2008) Deep surveying of alternative splicing complexity in the human transcriptome by highthroughput sequencing. Nat Genet 40:1413-1415. https://doi.org/10. 1038/ng.259

32. Pottier C, Hannequin D, Coutant S, Rovelet-Lecrux A, Wallon D, Rousseau S, Legallic S, Paquet C, Bombois S, Pariente J, Thomas-Anterion C, Michon A, Croisile B, Etcharry-Bouyx F, Berr C, Dartigues J-F, Amouyel P, Dauchel H, Boutoleau-Bretonniere C, Thauvin C, Frebourg T, Lambert J-C, Campion D (2012) High frequency of potentially pathogenic SORL1 mutations in autosomal dominant early-onset Alzheimer disease. Mol Psychiatry 17:875-879. https://doi.org/10.1038/mp.2012.15

33. Prudencio M, Belzil W, Batra R, Ross CA, Gendron TF, Pregent LI, Murray ME, Overstreet KK, Piazza-Johnston AE, Desaro P, Bieniek KF, DeTure M, Lee WC, Biendarra SM, Davis MD, Baker MC, Perkerson RB, van Blitterswijk M, Stetler CT, Rademakers R, Link CD, Dickson DW, Boylan KB, Li H, Petrucell $L$ (2015) Distinct brain transcriptome profiles in C9orf72-associated and sporadic ALS. Nat Neurosci 18:1175-1182. https://doi.org/10.1038/nn 4065

34. Raj B, Blencowe BJ (2015) Alternative splicing in the mammalian nervous system: recent insights into mechanisms and functional roles. Neuron 87:14-27. https://doi.org/10.1016/j.neuron.2015.05.004

35. Rockenstein EM, McConlogue L, Tan H, Power M, Masliah E, Mucke L (1995) Levels and alternative splicing of amyloid beta protein precursor (APP) transcripts in brains of APP transgenic mice and humans with Alzheimer's disease. J Biol Chem 270:28257-28267. https://doi.org/10.1074/ jbc.270.47.28257

36. Rogaeva E, Meng Y, Lee JH, Gu Y, Kawarai T, Zou F, Katayama T, Baldwin CT, Cheng R, Hasegawa H, Chen F, Shibata N, Lunetta KL, Pardossi-Piquard R, Bohm C, Wakutani Y, Cupples LA, Cuenco KT, Green RC, Pinessi L, Rainero I, Sorbi S, Bruni A, Duara R, Friedland RP, Inzelberg R, Hampe W, Bujo H, Song Y-Q, Andersen OM, Willnow TE, Graff-Radford N, Petersen RC, Dickson D, Der SD, Fraser PE, Schmitt-Ulms G, Younkin S, Mayeux R, Farrer LA, St George-Hyslop P (2007) The neuronal sortilin-related receptor SORL1 is genetically associated with Alzheimer disease. Nat Genet 39:168-177. https://doi.org/10.1038/ng1943

37. Sato $\mathrm{N}$, Hori O, Yamaguchi A, Lambert JC, Chartier-Harlin MC, Robinson PA, Delacourte A, Schmidt AM, Furuyama T, Imaizumi K, Tohyama M, Takagi T (1999) A novel presenilin-2 splice variant in human Alzheimer's disease brain tissue. J Neurochem 72:2498-2505. https://doi.org/10. 1046/j.1471-4159.1999.0722498.x

38. Scherzer CR, Offe K, Gearing M, Rees HD, Fang G, Heilman CJ, Schaller C, Bujo H, Levey Al, Lah JJ (2004) Loss of apolipoprotein E receptor LR11 in Alzheimer disease. Arch Neurol 61:1200-1205. https://doi.org/10.1001/ archneur.61.8.1200

39. Schmidt V, Sporbert A, Rohe M, Reimer T, Rehm A, Andersen OM, Willnow TE (2007) SorLA/LR1 1 regulates processing of amyloid precursor protein via interaction with adaptors GGA and PACS-1. J Biol Chem 282:3295632964. https://doi.org/10.1074/jbc.M705073200

40. Seo J, Singh NN, Ottesen EW, Lee BM, Singh RN (2016) A novel humanspecific splice isoform alters the critical C-terminus of Survival Motor Neuron protein. Sci Rep 6:30778. https://doi.org/10.1038/srep30778

41. Shabalin AA (2012) Matrix eQTL: ultra fast eQTL analysis via large matrix operations. Bioinformatics 28:1353-1358. https://doi.org/10.1093/bioin formatics/bts 163

42. Tanaka M (2015) The dendritic differentiation of Purkinje neurons: unsolved mystery in formation of unique dendrites. Cerebellum 14:227-230. https://doi.org/10.1007/s12311-014-0585-0

43. Tushev G, Glock C, Heumuller M, Biever A, Jovanovic M, Schuman EM (2018) Alternative $3^{\prime}$ UTRs modify the localization, regulatory potential, stability, and plasticity of mRNAs in neuronal compartments. Neuron 98:495-511.e6. https://doi.org/10.1016/j.neuron.2018.03.030

44. Verheijen J, Van den Bossche T, van der Zee J, Engelborghs S, SanchezValle R, Llado A, Graff C, Thonberg H, Pastor P, Ortega-Cubero S, Pastor MA, Benussi L, Ghidoni R, Binetti G, Clarimon J, Lleo A, Fortea J, de Mendonca A, Martins M, Grau-Rivera O, Gelpi E, Bettens K, Mateiu L, Dillen L, Cras P, De Deyn PP, Van Broeckhoven C, Sleegers K (2016) A comprehensive study of the genetic impact of rare variants in SORL1 in European early-onset Alzheimer's disease. Acta Neuropathol 132:213-224. https:// doi.org/10.1007/s00401-016-1566-9

45. Vuong CK, Black DL, Zheng S (2016) The neurogenetics of alternative splicing. Nat Rev Neurosci 17:265-281. https://doi.org/10.1038/nrn.2016. 27

46. Wang M, Beckmann ND, Roussos P, Wang E, Zhou X, Wang Q, Ming C, Neff R, Ma W, Fullard JF, Hauberg ME, Bendl J, Peters MA, Logsdon B, Wang P, Mahajan M, Mangravite LM, Dammer EB, Duong DM, Lah JJ, Seyfried NT, Levey Al, Buxbaum JD, Ehrlich M, Gandy S, Katsel P, Haroutunian V, Schadt E, Zhang B (2018) The Mount Sinai cohort of large-scale genomic, transcriptomic and proteomic data in Alzheimer's disease. Sci Data 5:180185. https://doi.org/10.1038/sdata.2018.185

47. Wang S, Wang B, Pan N, Fu L, Wang C, Song G, An J, Liu Z, Zhu W, Guan Y, XU Z-QD, Chan P, Chen Z, Zhang YA (2015) Differentiation of human induced pluripotent stem cells to mature functional Purkinje neurons. Sci Rep 5:9232. https://doi.org/10.1038/srep09232

48. Watson LM, Wong MMK, Vowles J, Cowley SA, Becker EBE (2018) A simplified method for generating purkinje cells from human-induced pluripotent stem cells. Cerebellum 17:419-427. https://doi.org/10.1007/ s12311-017-0913-2 
49. Young JE, Boulanger-Weill J, Williams DA, Woodruff G, Buen F, Revilla AC, Herrera C, Israel MA, Yuan SH, Edland SD, Goldstein LSB (2015) Elucidating molecular phenotypes caused by the SORL1 Alzheimer's disease genetic risk factor using human induced pluripotent stem cells. Cell Stem Cell 16:373-385. https://doi.org/10.1016/j.stem.2015.02.004

50. Zeng L, Zhang C, Xu J, Ye X, Wu Q, Dai J, Ji C, Gu S, Xie Y, Mao Y (2002) A novel splice variant of the cell adhesion molecule contactin 4 ( CNTN4) is mainly expressed in human brain. J Hum Genet 47:497-499. https://doi. org/10.1007/s100380200073

\section{Publisher's Note}

Springer Nature remains neutral with regard to jurisdictional claims in published maps and institutional affiliations.
Ready to submit your research? Choose BMC and benefit from:

- fast, convenient online submission

- thorough peer review by experienced researchers in your field

- rapid publication on acceptance

- support for research data, including large and complex data types

- gold Open Access which fosters wider collaboration and increased citations

- maximum visibility for your research: over $100 \mathrm{M}$ website views per year

At BMC, research is always in progress.

Learn more biomedcentral.com/submissions 